\title{
Climate SPHINX: evaluating the impact of resolution and stochastic physics parameterisations in the EC-Earth global climate model
}

\author{
Paolo Davini ${ }^{1,2}$, Jost von Hardenberg ${ }^{3}$, Susanna Corti ${ }^{2}$, Hannah M. Christensen ${ }^{4,5}$, Stephan Juricke ${ }^{4}$, \\ Aneesh Subramanian ${ }^{4}$, Peter A. G. Watson ${ }^{4}$, Antje Weisheimer ${ }^{4,6,7}$, and Tim N. Palmer ${ }^{4}$ \\ ${ }^{1}$ Laboratoire de Météorologie Dynamique/IPSL, Ecole Normale Supérieure, PSL Research University, CNRS, Paris, France \\ ${ }^{2}$ Institute of Atmospheric Sciences and Climate (ISAC-CNR), Bologna, Italy \\ ${ }^{3}$ Institute of Atmospheric Sciences and Climate (ISAC-CNR), Torino, Italy \\ ${ }^{4}$ Atmospheric, Oceanic and Planetary Physics, University of Oxford, Oxford, UK \\ ${ }^{5}$ National Center for Atmospheric Research (NCAR), Boulder, Colorado, USA \\ ${ }^{6}$ National Centre for Atmospheric Science (NCAS), University of Oxford, Oxford, UK \\ ${ }^{7}$ European Centre for Medium-Range Weather Forecasts (ECWMF), Reading, UK \\ Correspondence to: Paolo Davini (pdavini@lmd.ens.fr)
}

Received: 7 May 2016 - Discussion started: 23 June 2016

Revised: 17 February 2017 - Accepted: 26 February 2017 - Published: 31 March 2017

\begin{abstract}
The Climate SPHINX (Stochastic Physics HIgh resolutioN eXperiments) project is a comprehensive set of ensemble simulations aimed at evaluating the sensitivity of present and future climate to model resolution and stochastic parameterisation. The EC-Earth Earth system model is used to explore the impact of stochastic physics in a large ensemble of 30-year climate integrations at five different atmospheric horizontal resolutions (from 125 up to $16 \mathrm{~km}$ ). The project includes more than 120 simulations in both a historical scenario (1979-2008) and a climate change projection (2039-2068), together with coupled transient runs (1850-2100). A total of 20.4 million core hours have been used, made available from a single year grant from PRACE (the Partnership for Advanced Computing in Europe), and close to 1.5 PB of output data have been produced on SuperMUC IBM Petascale System at the Leibniz Supercomputing Centre (LRZ) in Garching, Germany. About 140 TB of postprocessed data are stored on the CINECA supercomputing centre archives and are freely accessible to the community thanks to an EUDAT data pilot project. This paper presents the technical and scientific set-up of the experiments, including the details on the forcing used for the simulations performed, defining the SPHINX v1.0 protocol. In addition, an overview of preliminary results is given. An improvement in the simulation of Euro-Atlantic atmospheric blocking following resolution increase is observed. It is also shown that
\end{abstract}

including stochastic parameterisation in the low-resolution runs helps to improve some aspects of the tropical climate - specifically the Madden-Julian Oscillation and the tropical rainfall variability. These findings show the importance of representing the impact of small-scale processes on the large-scale climate variability either explicitly (with highresolution simulations) or stochastically (in low-resolution simulations).

\section{Introduction}

The simulation and prediction of Earth's climate is one of the scientific and computational grand challenges. In order to make quantitative projections of future climate, it is necessary to use climate models that simulate all the important processes governing the evolution of the climate system. Over the past few decades, climate models have developed considerably - increasing both in complexity and resolution - as computational power has increased. Yet there is a notable difference in the horizontal resolution of models used in operational numerical weather prediction (NWP) and those used for climate simulations in the fifth Coupled Model Intercomparison Project (CMIP5; Taylor et al., 2012). Atmospheric horizontal resolutions in operational NWP are in the range 
of 16-40 km, whereas the resolution of CMIP5 climate models is (on average) coarser than $120 \mathrm{~km}$.

It is well known that a typical climate model is unable to represent many sub-synoptic-scale systems, and only poorly represents smaller baroclinic features. Typically climate models underestimate the number of observed storms (Zappa et al., 2013) and poorly simulate the statistics of atmospheric mid-latitude blocking (Davini and D'Andrea, 2016). In fact it has been shown (e.g. van Oldenborgh et al., 2012) that at standard (low) climate resolution, forecast systems have pervasive systematic errors, which impact on quasi-persistent weather regimes (Dawson et al., 2012) and, more generally, on temporal variability and regional patterns of the leading modes of variability (Delworth et al., 2012; Kinter III et al., 2013). On the other hand, recent experiments have shown that high-resolution climate models are significantly better at simulating important physical processes, such as the global water cycle (Demory et al., 2014), as well as relevant features of the large-scale atmospheric circulation such as the jet stream (Lu et al., 2015), the Euro-Atlantic blocking (Jung et al., 2012) and the Madden-Julian Oscillation (MJO; Peatman et al., 2015).

The fact that enhanced horizontal resolution in climate models can positively impact some aspects of the simulated large-scale atmospheric circulation is further evidence of the role that small-scale processes play in "shaping" large-scale motions. However, it is unlikely that climate integrations at very high resolution (i.e. at the resolution used in NWP), will be feasible in the near future. There are numerous other areas of climate model development that compete for the given computing resources, e.g. the need for ensembles of integrations, the need to integrate over century and longer timescales, and the need to incorporate additional Earth system complexity. In addition, parameterisations, which have been developed for coarse scales, may need retuning or to be replaced with alternative parameterisations at higher resolutions, which require a consistent development effort.

Instead of explicitly resolving small-scale processes by increasing the resolution of climate models, a possible alternative is to use stochastic parameterisation schemes. There has been significant progress in developing stochastic schemes over the last decade, primarily for use in medium-range and seasonal ensemble forecasts (e.g. Plant and Craig, 2008; Khouider et al., 2010; Bengtsson et al., 2013; Grell and Freitas, 2013; Dorrestijn et al., 2016; Sakradzija et al., 2016; Ollinaho et al., 2016). These schemes introduce an element of randomness into physical parameterisation schemes to account for the impact of uncertain, unresolved processes on the resolved-scale flow (Palmer, 2012). Stochastic schemes have been shown to improve the reliability of probabilistic forecasts on medium-range and seasonal timescales, as well as improving biases in the mean state.

There is mounting evidence that stochastic parameterisations can also prove beneficial for climate simulations (e.g. Lin and Neelin, 2000, 2003; Arnold et al., 2013). Berner et al.
(2012) showed that including stochastic physics can reduce systematic biases in the model's mean climate, comparable to improvements gained by increasing the model resolution. Several recent papers have also demonstrated that the variability of a climate model can significantly improve with the introduction of a stochastic physics scheme, with improvements observed in the representation of the MJO (Deng et al., 2015; Wang et al., 2016), the El Niño-Southern Oscillation (Christensen et al., 2017a) and extra-tropical flow regimes (Weisheimer et al., 2014; Dawson and Palmer, 2015; Christensen et al., 2015). As was the case for the mean state, the observed improvements can be similar to that observed on increasing the resolution of the model (Dawson et al., 2012).

These results highlight the influence of small-scale processes on large-scale climate variability, and indicate that although simulating variability at small scales is a necessity, it may not be necessary to represent the small scales accurately, or even explicitly, in order to improve the simulation of large-scale climate. This issue is important in light of the next CMIP6 project. In fact, it seems quite unrealistic that in the near future climate simulations at NWP resolution could be affordable. However, resolutions around $40 \mathrm{~km}$ might be more feasible and indeed they are planned within the HighResMIP project (Haarsma et al., 2016).

In the coordinated project Climate SPHINX (Stochastic Physics HIgh resolutioN eXperiments), we use the ECEarth Earth system model (Hazeleger et al., 2010, 2012, http://www.ec-earth.org) to investigate the sensitivity of climate simulations to model resolution and stochastic parameterisations. A key aim of the study is to investigate the degree to which stochastic parameterisation schemes can be used as a computationally cheaper alternative to increased model resolution.

The experiments follow one historical and one scenario projection following CMIP5 specifications in AMIP configuration (i.e. atmosphere-only integrations forced with observed - for the past - and simulated - for the future - sea surface temperatures). The AMIP integrations have been carried out keeping constant the vertical resolution (91 levels) and exploring five different horizontal resolutions: (i) low $(\sim 125 \mathrm{~km})$, (ii) moderate $(\sim 80 \mathrm{~km})$, (iii) intermediate $(\sim 40 \mathrm{~km})$, (iv) high $(\sim 25 \mathrm{~km})$ and (v) very high $(\sim 16 \mathrm{~km})$. Each integration is repeated with the joint implementation of two stochastic parameterisations: the Stochastically Perturbed Parameterisation Tendencies (SPPT) scheme (Palmer et al., 2009) and the Stochastic Kinetic Energy Backscatter (SKEB) scheme (Shutts, 2005; Palmer et al., 2009). In order to sample the natural variability, several ensemble members are produced for each configuration. The simulations (ii-iv) aim to investigate whether configurations with intermediate horizontal resolution are able to partially bridge the gap between very high and low resolution. In other words, a systematic comparison is useful to understand whether a gradual increase in resolution will lead to a similar gradual improvement of climate simulations or whether there 
is a true passing threshold in resolution, which is required to get acceptable simulations of the main climate features.

By comparing integrations carried out at different resolutions, we evaluate the impact of increased atmospheric horizontal resolution on the simulation of key climate processes and of climate variability. By comparing experiments with and without the implementation of stochastic physics, we evaluate the impact of stochastic physics on the simulation of key climate process and of the associated climate variability when the model resolution is the same. By comparing experiments with the implementation of stochastic physics with experiments carried out without stochastic physics, but at higher resolutions, we assess to what extent the stochastic representation of the sub-grid processes can compete with a more refined horizontal resolution. The results of this project integrate with several other efforts currently underway (e.g. the European Union's Horizon 2020 PRIMAVERA project, https://www.primavera-h2020.eu/). In particular this study complements groundbreaking past initiatives in pioneering the use of HPC (high-performance Computing) for climate simulations such as the UPSCALE (Mizielinski et al., 2014) and the ATHENA (Kinter III et al., 2013) projects.

Climate SPHINX was made possible by a considerable amount of computing time provided by PRACE (the Partnership for Advanced Computing in Europe) and data storage from EUDAT (the collaborative pan-European infrastructure providing research data services). We were granted 20 million core hours during a single year at SuperMUC, the IBM Petascale System at the Leibniz Supercomputing Centre (LRZ) in Garching near Munich, Germany. Storage of data produced by Climate SPHINX is secured by the EUDAT pilot project DATA SPHINX (DATA Storage and Preservation of High-resolution climate eXperiments), which provides a widely accessible archive for medium-term storage to facilitate data access and discovery. DATA SPHINX is managed by CINECA (the largest Italian computing centre) and at present hosts $140 \mathrm{~TB}$ of data generated by Climate SPHINX.

In this paper we describe in detail the important technical aspects of this project and highlight some preliminary scientific results on the impact of increased resolution and stochastic parameterisations on climate simulations. Model configuration and tuning are presented in Sect. 2, while the experimental set-up is described in Sect. 3. Section 4 is devoted to detail the technical configuration. An overview of results and concluding remarks are reported in Sects. 5 and 6, which is followed by the "Data availability" section.

\section{The EC-Earth global climate model}

In Climate SPHINX, version 3.1 of the state-of-the-art ECEarth atmosphere-ocean Earth system model (Hazeleger et al., 2010, 2012) has been used.

The atmospheric component of EC-Earth is based on cycle $36 r 4$ of the Integrated Forecast System (IFS) circula- tion model (ECWMF, 2009), which has been developed by the European Centre for Medium-Range Weather Forecasts (ECMWF). This has been tuned and improved for climate purposes by the EC-Earth consortium. IFS uses a combination of spectral and reduced Gaussian grids (where, in the latter, the number of longitudinal grid points decreases towards the poles). Physical parameterisations and advection are computed on the reduced Gaussian grid and then, using the spectral transform, semi-implicit time stepping is performed in the spectral space.

Traditionally, the spectral harmonic at which truncation occurs defines the horizontal resolution; IFS uses a linear triangular truncation for which a specified number of $\mathrm{N}$ harmonics retained corresponds to $2(N+1)$ grid points along the Equator. If the resolution is T255, this means that postprocessed output will have $512 \times 256$ grid points on a regular Gaussian grid, which corresponds to a resolution of about $80 \mathrm{~km}$ at the Equator. The description of the main parameterisation schemes within IFS can be found in Beljaars et al. (2004); the parameterisations are in general independent of resolution, with the only exception of the convective adjustment time, which decreases with increasing resolution as reported in Table 1.

To represent land-surface dynamics, IFS integrates the Hydrology Tiled ECMWF Scheme of Surface Exchanges over Land (H-TESSEL) land-surface scheme (Balsamo et al., 2009). When used in coupled mode, the Nucleus for European Modelling of the Ocean (NEMO) version 3.3.1 oceanic circulation model (Madec, 2008) is used; this makes use of a tripolar grid with the poles placed over northern Siberia, North America and Antarctica. NEMO includes the Louvain la Neuve (LIM) sea ice model version 3 (Vancoppenolle et al., 2012). The atmospheric and oceanic components are coupled through OASIS3 (Valcke, 2013), with a coupling frequency of $3 \mathrm{~h}$.

\subsection{The stochastic physics parameterisation schemes}

We consider two complementary approaches to stochastic parameterisation, both developed at ECMWF for IFS. The two schemes considered here are used operationally at weather and seasonal forecasting centres worldwide (Palmer et al., 2009; Yonehara and Ujiie, 2011; Bouttier et al., 2012; Sanchez et al., 2016), and so have been extensively tested in a range of models on medium range and seasonal timescales. Following the "seamless prediction" paradigm, we choose to test these schemes here on climate timescales.

The first approach is the SPPT scheme (Palmer et al., 2009), which uses multiplicative noise to represent model uncertainty due to the parameterisation process. The use of multiplicative noise has been motivated through several coarse-graining studies (Shutts and Palmer, 2007; 
Table 1. Resolution-dependent scientific configuration for EC-Earth in the Climate SPHINX experiments. The same number of ensemble members has been run for present-day AMIP (PDA) and future scenario AMIP (FSA) experiments. T255C is the coupled configuration used for past-to-future coupled (PFC) simulations. Resolution is estimated at the Equator. The number of members indicate the deterministic and stochastic members. Backscatter ratio (tuning parameter for SKEB stochastic scheme), convective adjustment time (tuning parameter for deep convection) and momentum launch (tuning parameter for non-orographic gravity waves) are unitless.

\begin{tabular}{lrrrrrr}
\hline Truncation & Resolution & No. of members & Time step & Backscatter ratio & Conv. adj. time & Mom. launch \\
\hline T159 & $125.2 \mathrm{~km}$ & $10+10$ & $3600 \mathrm{~s}$ & 0.032 & 2.6 & 0.00375 \\
T255 & $78.3 \mathrm{~km}$ & $10+10$ & $2700 \mathrm{~s}$ & 0.040 & 2.0 & 0.00375 \\
T511 & $39.1 \mathrm{~km}$ & $6+6$ & $900 \mathrm{~s}$ & 0.085 & 1.5 & 0.00375 \\
T799 & $25.0 \mathrm{~km}$ & $3+3$ & $720 \mathrm{~s}$ & 0.095 & 1.3 & 0.00368 \\
T1279 & $15.7 \mathrm{~km}$ & $1+1$ & $600 \mathrm{~s}$ & 0.095 & 1.2 & 0.00334 \\
\hline T255C & $78.3 \mathrm{~km}$ & $3+3$ & $2700 \mathrm{~s}$ & 0.040 & 2.0 & 0.00375 \\
\hline
\end{tabular}

Shutts and Pallarès, 2014). SPPT can be expressed as

$$
\frac{\partial X}{\partial t}=D+K+(1+\mu e) \sum_{i} P_{i}
$$

where $\frac{\partial X}{\partial t}$ is the modelled total tendency in $X$. This is the sum of $D$, the dynamical tendency, $K$, the horizontal diffusion, and each $P_{i}$ term, with $P_{i}$ being the tendency from the $i$ th physics scheme. The zero mean random perturbation, $e$, is constant in the vertical, but $\mu$ tapers the perturbation to zero close to the surface and in the stratosphere. The scheme acts on the tendencies of the physical fields (i.e. temperature, winds and specific humidity) resulting from the five main parameterisation schemes: radiation, turbulence and gravity wave drag, non-orographic gravity wave drag, convection, and large-scale water processes. All variables are perturbed with the same random number.

The perturbation, $e$, is generated using a spectral pattern generator (Berner et al., 2009), ensuring that it smoothly varies in space, while the patterns evolve in time following an $\operatorname{AR}(1)$ process. The perturbation at each time step is the sum of three independent random fields, which represent uncertainties on different temporal and spatial scales. The fields have horizontal correlations of 500, 1000 and $2000 \mathrm{~km}$ and temporal decorrelations of $6 \mathrm{~h}, 3$ days and 30 days respectively, with associated standard deviations of $0.52,0.18$ and 0.06 . The magnitude of the perturbation has been motivated through coarse-graining high-resolution model simulations (Shutts and Pallarès, 2014), and a recent coarsegraining study has also provided justification for the noise temporal and spatial correlation scales (Christensen et al., $2017 \mathrm{~b})$. While the smallest scale $(500 \mathrm{~km}$ and $6 \mathrm{~h})$ dominates on weather forecasting timescales, it is expected that the larger scales will also be important on climate timescales. The SPPT scheme requires no retuning with changing horizontal resolution: the same noise characteristics are used operationally at ECMWF across model resolutions. This is because the multiplicative nature of the scheme applied to the total of all parameterised physical tendencies results in perturbations to the model that scale automatically as the pa- rameterised tendencies scale with resolution. The resolution dependence of the individual contributions from the parameterisation schemes is implicitly dealt with at the (deterministic) parameterisation level.

In contrast to SPPT, the SKEB scheme aims to represent a physical process that is otherwise absent from the model (Shutts, 2005; Palmer et al., 2009). Kinetic energy loss is common in models due to numerical integration schemes and the parameterisation process (Berner et al., 2009). To counteract this, the SKEB scheme represents upscale transfer of kinetic energy by randomly perturbing the stream function.

Similar to SPPT, the SKEB scheme uses a spectral pattern generator to generate a spatially and temporally correlated perturbation field, which is added at each time step to the deterministic stream function tendency,

$\dot{\psi}_{\mathrm{t}}(\phi, \lambda, t)=\dot{\psi_{\operatorname{det}}}(\phi, \lambda, t)+f(\phi, \lambda, t)$,

where $\dot{\psi}_{\mathrm{t}}$ is the total stream function tendency, $\dot{\psi}_{\operatorname{det}}$ is the deterministic tendency and $f$ is the additive perturbation field. The perturbation field is expressed in spherical harmonics, and each coefficient is evolved separately in time following an AR(1) process. A tuning parameter for the SKEB scheme, the backscatter ratio, is set to increase following resolution increase (see Table 1; following practice at ECMWF) in order to improve the slope of the kinetic energy spectrum.

The standard SPPT and SKEB schemes were designed for use at NWP timescales. When implemented in the EC-Earth climate model, it was found that the SPPT scheme resulted in a large imbalance involving the water cycle, with a negative precipitation minus evaporation $(P-E)$ that was 10 times larger than in the deterministic model associated with an anomalous latent heat flux and a negative net surface flux of about $2 \mathrm{~W} \mathrm{~m}^{-2}$. This arises because the SPPT scheme was not designed specifically to conserve water vapour, and resulted in a water vapour sink in the atmosphere. A fix has been implemented, requiring that the global average of the tendencies (i.e. winds, temperature and more importantly specific humidity) before and after the SPPT perturbation is conserved. The new scheme removes the imbalance in $P-E$, 
which is now equal to that in the run where the SPPT scheme is disabled. This fix has subsequently been implemented at ECMWF (Leutbecher et al., 2017).

Hereafter, Climate SPHINX simulations where stochastic parameterisation is operational will be defined as "stochastic" runs, while simulations where the scheme is deactivated will be mentioned as "deterministic" runs.

\subsection{Model tuning}

With respect to the previous version (v3.0.1), EC-Earth 3.1 shows a reduced radiative imbalance and an improved hydrological cycle (Davini et al., 2014). However, it still exhibits a cold bias in both its atmosphere-only and coupled configuration and a small imbalance in $P-E$. In a "present day" AMIP configuration, the 3.1 version is too cold, extracting heat from the underlying sea surface temperatures (SSTs) by about $1.5 \mathrm{~W} \mathrm{~m}^{-2}$ and showing unrealistically high values for net SW (shortwave) and LW (longwave) fluxes at TOA (top of the atmosphere) (around 243-244 $\mathrm{W} \mathrm{m}^{-2}$ ). Thus, the first goal of the tuning was to provide reasonable radiative fluxes at TOA and at the surface for the standard deterministic version of the model (T255; see next paragraph for further description on the configurations adopted).

To improve the radiation budget, some of the convection and microphysical parameters from a more recent version of IFS (cy40r1) were retrieved. In addition to this, two standard tuning parameters have been modified (see Mauritsen et al., 2012); the entrainment rate for organised convection (ENTRORG) was reduced from $1.75 \times 10^{-4}$ to $1.5 \times 10^{-4}$, and the rate of conversion of liquid water to rain (RPRCON) was reduced from $1.4 \times 10^{-3}$ to $1.2 \times 10^{-3}$.

The optimal choice of tuning parameters provides reasonable fluxes at the TOA (around $240 \mathrm{~W} \mathrm{~m}^{-2}$ ) and a positive flux at the surface of about $0.6 \mathrm{~W} \mathrm{~m}^{-2}$, in accordance with the best estimates from observations (Wild et al., 2013). It is important to note that the tuning of the radiative fluxes has been carried out only for the T255 deterministic model version: the radiative balance has not been tuned in the higher resolution or stochastic models. This ensures a clean comparison between simulations at different resolutions and with and without stochastic physics. If the model were re-tuned for each run, it is not possible to determine whether it is changing the tuning parameters, changing the resolution or including stochastic physics that are responsible (Haarsma et al., 2016). Including the SPPT scheme led to a negative bias in the surface heat fluxes of about $0.8 \mathrm{~W} \mathrm{~m}^{-2}$, likely caused by a different distribution of the clouds.

The main radiative fluxes resulting after the complete tuning procedure are reported in Table 2. As shown in this table, the radiative balance of the model at higher resolution (and with stochastic physics) shows larger TOA SW and LW with increasing resolution. Net surface fluxes are highly variable, with higher values for coarser resolutions.
Table 2. Radiative fluxes expressed in $\mathrm{W} \mathrm{m}^{-2}$ for the reference experiment (i.e. the first simulation run) at different resolution for present-day AMIP (PDA) simulations. D stands for deterministic simulation, $\mathrm{S}$ for stochastic. Fluxes have been tuned for T255D.

\begin{tabular}{lrrrr}
\hline Simulation & Net Sfc & Net TOA & TOA SW & TOA LW \\
\hline T159D & 1.57 & 1.22 & 239.93 & 238.71 \\
T159S & 0.75 & 0.33 & 239.32 & 238.99 \\
T255D & 0.67 & 0.41 & 240.23 & 239.82 \\
T255S & -0.16 & -0.49 & 239.65 & 240.14 \\
T511D & 0.16 & 1.05 & 241.50 & 240.44 \\
T511S & -0.75 & 0.19 & 241.07 & 240.88 \\
T799D & -0.12 & 1.16 & 242.10 & 240.94 \\
T799S & -0.82 & 0.47 & 241.78 & 241.31 \\
T1279D & -0.09 & 1.44 & 242.58 & 241.14 \\
T1279S & -1.09 & 0.41 & 242.16 & 241.74 \\
\hline
\end{tabular}

Sfc: surface; TOA: top of the atmosphere; SW: shortwave; LW: longwave.

Finally, a supplementary modification - derived from a more recent IFS cycle - has been performed in order to produce a realistic quasi-biennial oscillation (QBO) at all resolutions. The EC-Earth 3.1 non-orographic gravity waves scheme is characterised by a momentum flux that is continuously launched in the mid-troposphere to simulate the effect of gravity waves. The latitudinal profile of this momentum flux governs the correct parameterisation of gravity waves: a too high amplitude of the momentum flux will disturb the QBO in equatorial zones, particularly at high resolutions, while a too low value will lead to unrealistic eddydriven jets, especially in the Southern Hemisphere, where orographically induced wave drag is low. With the current latitudinal profile, the QBO was simulated only at standard resolution (T255 with 91 vertical levels). Following advice from ECMWF staff, a resolution-dependent parameterisation of non-orographic gravity wave drag replaced the versiondependent parameterisation present in EC-Earth 3.1 (an ad hoc parameterisation developed for the ECMWF System 4 seasonal forecast system). Namely, instead of using a lowmomentum flux average value $($ GFLUXLAUN $=0.02)$ with a positive Gaussian peak at $50^{\circ} \mathrm{S}$, we use a higher value (GFLUXLAUN $=0.0375$ ), which is reduced with a Gaussian shape at the Equator. This negative peak is slightly deeper for stochastic runs than for deterministic simulations to compensate for the effect of the stochastic noise. The average value of the momentum flux was further reduced with increasing resolution (starting from T799) according to the ECMWF specification for IFS cy40r1 (see Table 1).

The new non-orographic gravity wave scheme - a standard in the current operational forecast ECMWF model - allows for the simulation of the QBO at all the resolutions explored in Climate SPHINX, without deteriorating the jet streams. Given these positive results, the new parameterisation will be implemented in the upcoming EC-Earth 3.2 version. 


\section{Science configuration: the SPHINX v1.0 protocol}

The following sections describe the scientific configuration, including the simulations performed, the initial and boundary conditions, the SST and sea ice concentration (SIC) used that together define the SPHINX v1.0 protocol.

\subsection{Climate SPHINX simulations}

Climate SPHINX simulations are grouped into three main blocks: present-day AMIP (PDA), future scenario AMIP (FSA) and past-to-future coupled (PFC). PDA and FSA are atmosphere-only simulations: 20 ensemble members are run at T159 $(\sim 125 \mathrm{~km}), 20$ at T255 $(\sim 80 \mathrm{~km}), 12$ at T511 $(\sim 40 \mathrm{~km}), 6$ at T799 $(\sim 25 \mathrm{~km})$ and 2 at T1279 $(\sim 16 \mathrm{~km})$ for both PDA and FSA experiments. For each resolution, half of the ensemble members have the stochastic physics parameterisations activated. All simulations have the same vertical grid with 91 levels (L91): these are hybrid levels with the last full level at $0.01 \mathrm{hPa}$. The number of ensemble members run and their resolution are also reported in Table 1.

The atmosphere-only experiments extend for 30 consecutive years, from 1979 to 2008 for PDA, while FSA experiments are run from 2039 to 2068.

PFC simulations are run with IFS at the T255L91 configuration, coupled with NEMO using the ORCA1 grid (a tripolar grid with resolution of $1^{\circ}$ longitudinally and refinement to $1 / 3^{\circ}$ at the Equator) with 46 vertical levels. The upper model level is at ca. $3 \mathrm{~m}$ and 10 levels are in the upper $100 \mathrm{~m}$. Six ensemble members are run, three with the stochastic parameterisation active and three control members without stochastic parameterisation, from 1850 to 2100 .

\subsection{Initial conditions}

The initial conditions (ICs) in both the PDA and FSA experiments are taken from the ECMWF ERA-Interim Reanalysis (Dee et al., 2011) for 1 January 1979. A first experiment is run at each resolution for a few days, and it is used to create the ICs for the other experiments. For instance, for T255 experiments, the ICs for the 10 ensemble members are extracted using the midnight values (00:00) from each of the first 10 days respectively, and then reassigned to 1 January.

The same ICs are used also for FSA: in order to account for the land-surface adjustment to the new forcing, a 1-year spinup has been carried out for FSA (which is therefore starting from 2038).

For PFC simulations, given the different expected climatologies of integrations with/without stochastic physics, two 320 -year spin-ups are carried out in coupled mode to equilibrate the ocean to the atmospheric forcing. Having spun-up, three oceanic states - from spin-up year 300, 310 and $320-$ are coupled with three different atmospheric ICs; these are run in coupled mode for a further 10 years with fixed greenhouse gas (GHG) forcing for the year 1850. In this way the phase space distance between the simulations is 20 years and the atmosphere and land surface have had enough time to adjust to the new oceanic state.

\subsection{Forcing and boundary conditions}

Well-mixed GHGs, stratospheric ozone and volcanic aerosol concentrations have been set according to the CMIP5 protocol (Moss et al., 2010; Taylor et al., 2012). Historical forcing is used for PDA experiments, whereas for the FSA experiments the high emission scenario (Representative Concentration Pathway 8.5, RCP8.5) is adopted. PFC simulations use the historical CMIP5 specification from year 1850 to year 2005 included; after that, the forcing is taken from the RCP8.5 scenario. Albedo, land use and vegetation patterns are set using the standard configuration of EC-Earth 3.1, which uses a MODIS-derived fixed climatological seasonal cycle for snow-free albedo and the leaf area index. The average yearly solar irradiance was set at $1368.2 \mathrm{~W} \mathrm{~m}^{-2}$ with intrannual variations, following the standard EC-Earth 3.1 set-up. All the simulations of PDA and FSA experiments use this set-up. For the PFC simulations interannual variations following CMIP5 prescriptions (i.e. the 11-year solar cycle) have been added.

\subsection{Present-day SST and SIC}

Given that both FSA and PDA simulations are atmosphereonly runs, a special effort has been taken to provide reliable SSTs in order to fully exploit the high resolution.

For PDA, SSTs have been obtained from the daily SST and SIC HadISST2.1.1, a pentad-based dataset with a resolution of $0.25^{\circ} \times 0.25^{\circ}$ for SSTs (Kennedy et al., 2017) and $1^{\circ} \times 1^{\circ}$ for SIC (Titchner and Rayner, 2014). These are bilinearly interpolated onto the required reduced Gaussian grid for each resolution: climatologies for SST and SIC for the 1979-2008 period can be seen in the upper panels of Fig. 1 .

A number of inconsistencies are found between the landsea mask of IFS and of HadISST2.1.1; these are due to slightly different coastlines and a different representation of the lakes. For the different coastlines, linear extrapolation from HadISST2.1.1 has been performed. For the interior (i.e. lakes), a methodology similar to the one used in ERA20CM dataset (Hersbach et al., 2015) has been adopted: 1-month lagged $2 \mathrm{~m}$ temperatures from the ERA-Interim monthly climatology of 1979-2008 are used as SST. Where the temperature is below zero, SIC is set to one, otherwise it is left at zero. This is interpolated in time on a daily basis and in space on the needed grid to create a smoothed seasonal cycle for lakes.

\subsection{Future scenario SST and SIC}

The creation of SST and SIC for the FSA experiment is more complex. We would like to consider the mean change and trend for the future climate (2038-2068) predicted by the 


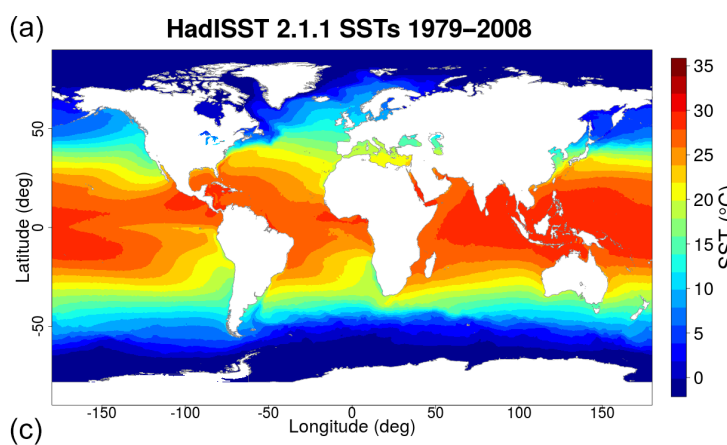

Future SSTs (2039-2068) minus HadISST2.1.1 (1979-2008)

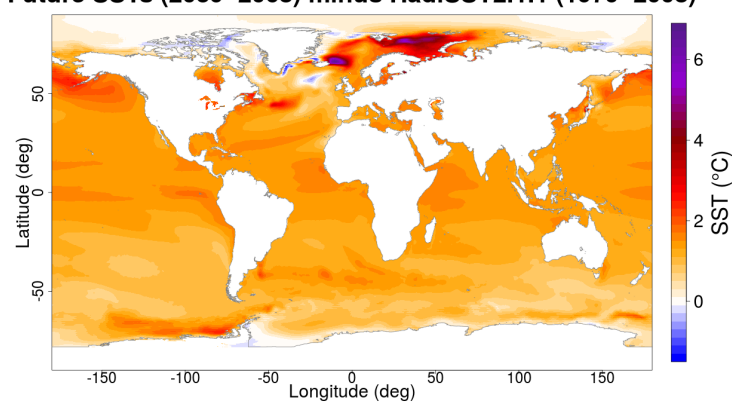

(b)

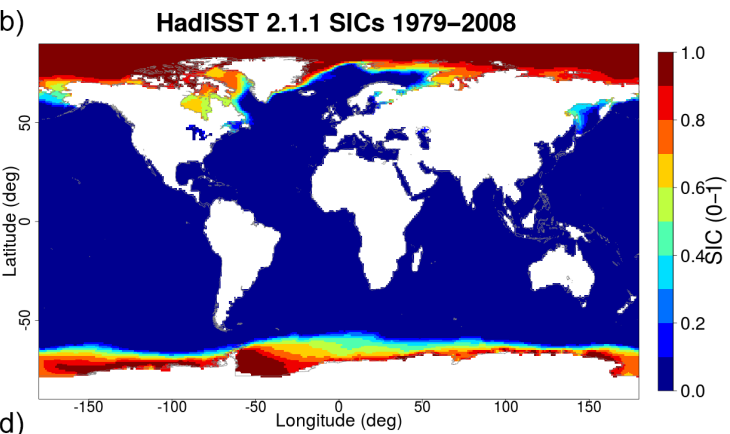

Future SICs (2039-2068) minus HadISST2.1.1 (1979-2008)

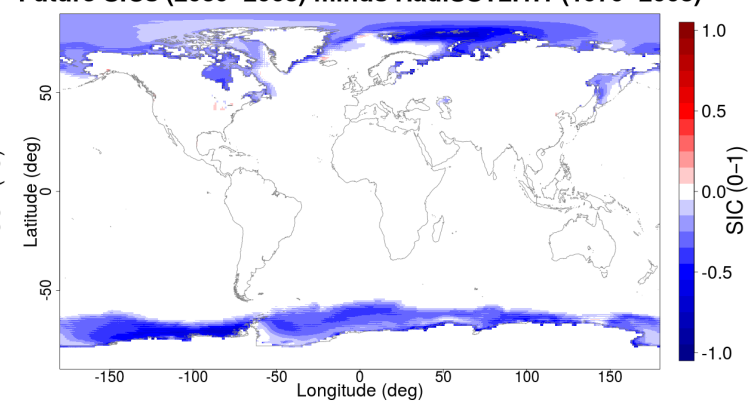

Figure 1. (a, b) HadISST 2.1.1 climatology for the SSTs (a) and SIC (b) for the 1979-2008 period. (c, d) Climatological changes between the FutureHadISST 2.1.1 dataset and the HadISST 2.1.1 dataset for SST (c) and SIC (d).

state-of-the-art global coupled models (i.e. the CMIP5 models). However, the oceanic component of these models has generally a low horizontal (of the order of $1^{\circ}$ ) and temporal (usually monthly) resolution, in which the oceanic circulation is not perfectly resolved. In order to improve our boundary conditions, we decided to take advantage of the high temporal and spatial resolution provided by the HadISST2.1.1. As a consequence, the SST for FSA experiments have been obtained as a combination of HadISST2.1.1 variability and the CMIP5 EC-Earth simulations ensemble mean trend.

First, the 1979-2008 HadISST2.1.1 SST has been detrended point by point to provide a set of anomalies with realistic variability. Second, the monthly seasonal cycle of the difference between the CMIP5 EC-Earth RCP8.5 ensemble mean over 2038-2068 (10 members) and the CMIP5 ECEarth historical ensemble mean over 1979-2008 (10 members) has been computed. This provides for each grid point the average expected SST increase from the present-day to the future period according to a GCM (Global Circulation Model), as a function of calendar month. To account for changes in SST during the FSA period, for each grid point the average trend in SST from the CMIP5 EC-Earth RCP8.5 integrations for 2038-2068 was also extracted. All CMIP5 EC-Earth data were bilinearly interpolated in space on the HadISST2.1.1 grid and linearly in time to daily frequency.

Finally, a new Step1HadISST dataset has been created combining the detrended HadISST2.1.1 (expressing the high-resolution daily variability), the average daily change of CMIP5 EC-Earth (from RCP8.5 and historical, express- ing the expected average temperature increase) and the linear trend of the CMIP5 EC-Earth RCP 8.5 (expressing the expected future trend in SST). The methodology used here, which shares the main characteristics with the method developed by Mizuta et al. (2008), is sketched in Fig. 2.

However, the Step1HadISST reconstruction misses an important element; there is no information on the sea ice cover in the future. To account for this, we took data from the CMIP5 EC-Earth simulations as a reference for SIC. CMIP5 EC-Earth simulations show a considerable cold bias in SST with respect to HadISST2.1.1, but they show good sea ice coverage, especially for the Northern Hemisphere (see average Northern Hemisphere and Southern Hemisphere SIC in Fig. 3).

Considering that an ensemble mean would be unrealistic, especially for a field with a large spatial variance as sea ice, we select a single ensemble member representative of the ensemble. Member "r8ilp1" has been chosen to characterise the ensemble, since its climatology shows the smallest SIC root mean square error (RMSE) when compared to the ensemble mean climatology in the time window 2038-2068. Clearly, using RMSE is only one of the possible metric to perform such selection; our main goal is to pick an ensemble member that is not an outlier when compared to the other EC-Earth CMIP5 ensemble members.

As a last step, we must evaluate SST for points where SIC coverage has disappeared in the future scenario. The lack of information about historical SST under sea ice results in undefined SST at these points using the methodology outlined 


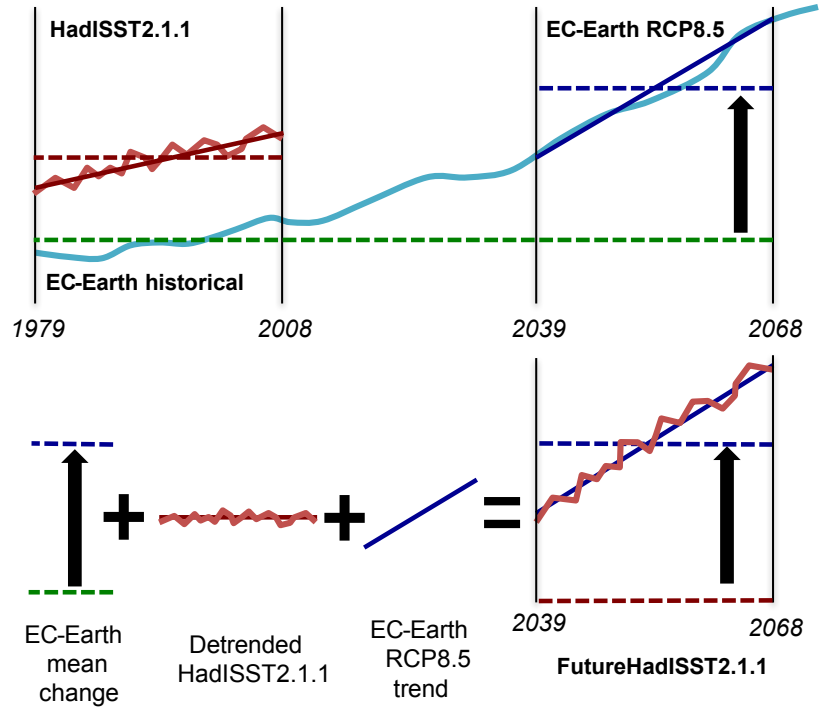

Figure 2. Scheme representing the methodology adopted to create the FutureHadISST 2.1.1. The new dataset is a combination of detrended daily variability from HadISST 2.1.1, CMIP5 EC-Earth mean change and CMIP5 EC-Earth RCP8.5 trend.

above. We define these as "bare point". For bare points, we want to make use of the model variability, but we do not want to have inconsistent SST at the boundaries (i.e. where bare points border the Step1Hadisst dataset).

Initially, we perform a linear extrapolation for bare points for Step1HadISST SSTs - which gives us a measure of the average SSTs at the bare points. However, these extrapolated values are missing a realistic spatial variability. We then mask the bare points also in the SST field of the CMIP5 ECEarth ensemble member "r8i1p1", and we subsequently linearly extrapolate new values. We then subtract from the original field of CMIP5 EC-Earth ensemble member "r8i1p1" these new extrapolated values, in order to obtain an anomaly field, which includes the spatial and temporal variability of the SST field over the bare points given by CMIP5 EC-Earth "r8ilp1". This final field is then added to the linearly extrapolated Step1HadISST SST.

Hence, for each day, the SSTs for bare points are given by the EC-Earth CMIP5 RCP8.5 ensemble member "r8i1p1" SST minus extrapolated EC-Earth CMIP5 RCP8.5 SSTs plus extrapolated Step1HadISST. The methodology to obtain this specific SST reconstruction is illustrated in Fig. 4.

This provides a pattern of SSTs physically consistent with SICs; indeed, it avoids unrealistic values of SSTs in the proximity of the polar cap during winter and - using the CMIP5 EC-Earth data - it provides a reasonable distribution of SSTs in summer, where in the future scenario the sea ice coverage in the Northern Hemisphere often disappears. Moreover, there is no discontinuity at the border with Step1HadISST. The new dataset is defined as FutureHadISST2.1.1.
The same methodology used for the PDA simulations has been adopted also for FSA runs in order to solve the issues of the lakes and the different land sea mask; however, in this case we must account for the estimated temperature change over land. We consider the difference between the 1-month lagged $2 \mathrm{~m}$ surface temperature from CMIP5 ECEarth RCP8.5 and the 1-month lagged $2 \mathrm{~m}$ surface temperature from CMIP5 EC-Earth historical ensemble (averaged over eight members). We then add this to the 1-month lagged $2 \mathrm{~m}$ surface temperature ERA-Interim monthly climatology of 1979-2008. This, analogous to what was done for SSTs, accounts for climate change.

The SST and SIC changes between FutureHadISST2.1.1 and HadISST2.1.1 are reported in Fig. 1; as expected larger warming and sea ice retreat is seen in the Northern Hemisphere high latitudes. Figure 3 reports the time series and trends for SST (between $45^{\circ} \mathrm{S}$ and $45^{\circ} \mathrm{N}$ ) and SIC (for both Northern and Southern hemispheres) for both FutureHadISST2.1.1 and HadISST2.1.1.

\section{Technical configuration}

\subsection{High-performance computing details}

Simulations have been run on the 6.8 PFLOP SuperMUC IBM Petascale System at LRZ. The initial set-up and configurations have been performed on the Supermuc-I platform, based on Sandy Bridge-EP Xeon E5-2680 8C processors. For processor decomposition the Message Passage Interface (MPI) parallelism paradigm has been used. EC-Earth allows also for OpenMP/Shared memory parallelisation, which has been tested without showing any significant computational benefit.

An accurate scaling of the performance was performed during the first months of the simulations. However, a conservative choice has been undertaken, after considering that the wall time needed to run the simulations was not the main concern for the project success. The number of cores assigned to each experiments have been selected following the resolution of the model considered. Although stochastic physics experiments showed about 5-10\% decrease in performance (according to different resolutions), the same number of cores has been retained.

In summer 2015, a new Supermuc-II platform based on Haswell Xeon E5-2697 v3 processors was made available by the LRZ. The new HPC granted a reduction of about $5 \%$ of the total core hours used, without affecting the wall time. About $75 \%$ of the simulations have been run using the Haswell nodes. Details on the processor decomposition, computational costs and data outputs are reported in Table 3.

\subsection{Data output and post-processing}

In Climate SPHINX, IFS has been set up to provide output in GRIB format every $3 \mathrm{~h}$; however, the four T1279 simu- 
(a) $\quad 45^{\circ} \mathrm{S}-45^{\circ} \mathrm{N}$ yearly averaged SSTs

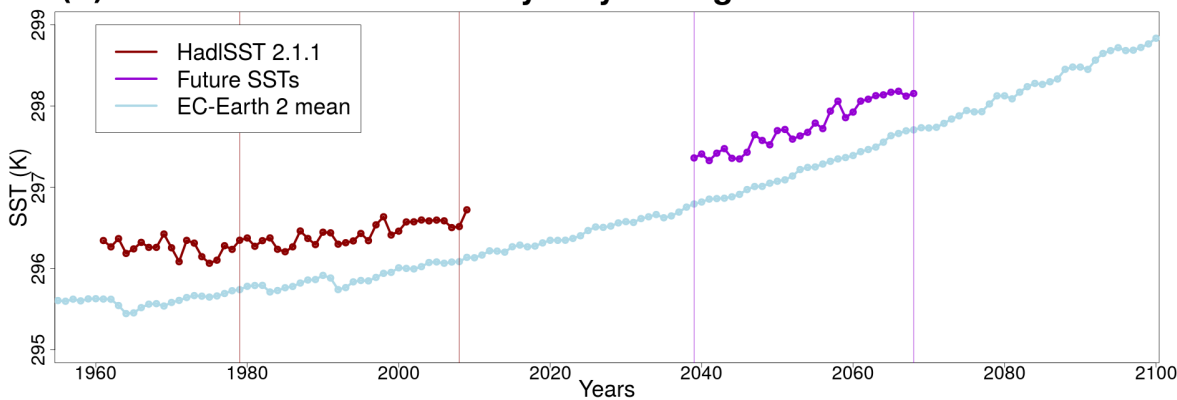

(b) Northern and Southern Hemisphere yearly averaged ice area

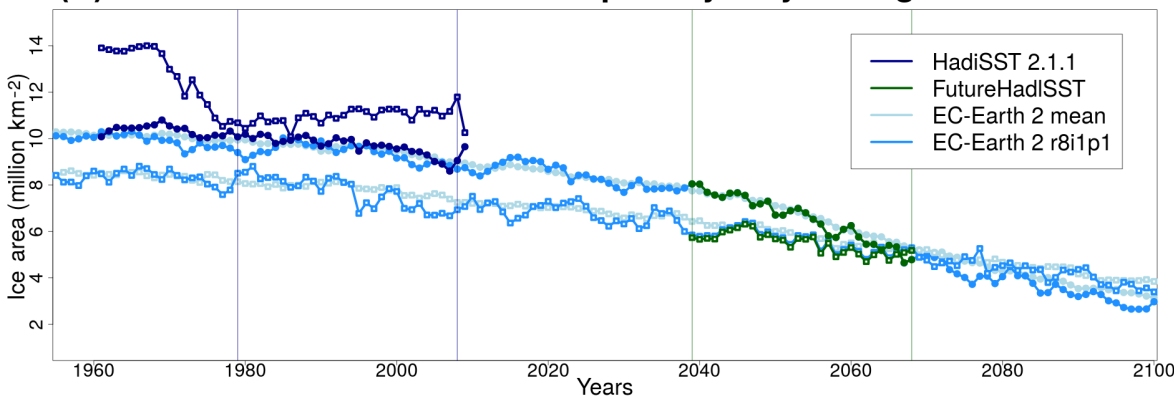

Figure 3. (a) Time series for $45^{\circ} \mathrm{S}-45^{\circ} \mathrm{N}$ yearly averaged SST for present-day HadISST 2.1 .1 (red), FutureHadISST 2.1 .1 (violet) and CMIP5 EC-Earth ensemble mean (light blue). (b) Time series for Northern Hemisphere (filled circles) and Southern Hemisphere (empty circles) yearly averaged sea ice area for HadISST 2.1.1 (dark blue), FutureHadISST 2.1.1 (green), CMIP5 EC-Earth ensemble member "r8i1p1" (light blue) and the CMIP5 EC-Earth ensemble mean (faint blue).

Table 3. Resolution-dependent technical details for EC-Earth in the Climate SPHINX experiments. T255C is the coupled configuration used for PFC simulations. Wall time has been measured on the Supermuc-II Haswell platform, and it is evaluated for deterministic simulations; stochastic simulations wall time is about the $5 \%$ higher.

\begin{tabular}{lrllrr}
\hline Truncation & No. of cores & Wall time (per year) & Leg length & Output data (per year) & Post-proc data (per year) \\
\hline T159 & 224 & $52 \mathrm{~min}$ & 1 year & $26 \mathrm{~GB}$ & $9.7 \mathrm{~GB}$ \\
T255 & 588 & $1 \mathrm{~h} 12 \mathrm{~min}$ & 1 year & $64 \mathrm{~GB}$ & $24 \mathrm{~GB}$ \\
T511 & 840 & $6 \mathrm{~h} 10 \mathrm{~min}$ & 6 months & $249 \mathrm{~GB}$ & $35 \mathrm{~GB}$ \\
T799 & 1120 & $14 \mathrm{~h}$ & 2 months & $605 \mathrm{~GB}$ & $57 \mathrm{~GB}$ \\
T1279 & 1540 & $30 \mathrm{~h}$ & 1 month & $1.6 \mathrm{~TB}$ & $111 \mathrm{~GB}$ \\
\hline T255C & 588 & $1 \mathrm{~h} 35 \mathrm{~min}$ & 1 year & $38 \mathrm{~GB}$ & $30 \mathrm{~GB}$ \\
\hline
\end{tabular}

lations alone sum up to about $200 \mathrm{~TB}$ of raw data output. Summing together the restarts files and the output of all experiments the total amount of space occupied at the peak of the project (February 2016) reached about $1 \mathrm{~PB}$. In order to reduce the size of the output and to increase the data accessibility to a larger audience, automatic post-processing routines have been implemented. At the end of each simulation leg, a script aimed at post-processing is launched; the script handles both the spectral and reduced Gaussian data from IFS and extracts and converts the requested variables from the default ECMWF format to a user-friendly, CMOR-like format on regular Gaussian grid. With this automatic procedure, more than $140 \mathrm{~TB}$ of post-processed data has been produced. A significant reduction of the data volume was obtained making use of the NetCDF-4 Zip format.

Monthly (MON), daily (DAY) and $6 \mathrm{~h}$ (6HRS) data for different subsets of variables have been produced. More than 50 fields have been stored at monthly frequency. In order to further reduce the space requirements, daily and $6 \mathrm{~h}$ threedimensional (3-D) fields have been degraded to the spectral resolution of T255. Additional data at $3 \mathrm{~h}$ frequency have been stored for the Euro-Cordex domain (3HRS-CDX) and for a sub-domain including India, Tibet and Pakistan (3HRSITP). Total precipitation has also been saved over the global grid at full resolution with $3 \mathrm{~h}$ frequency (3HRS). Finally, synoptic monthly means have been stored for the main radia- 


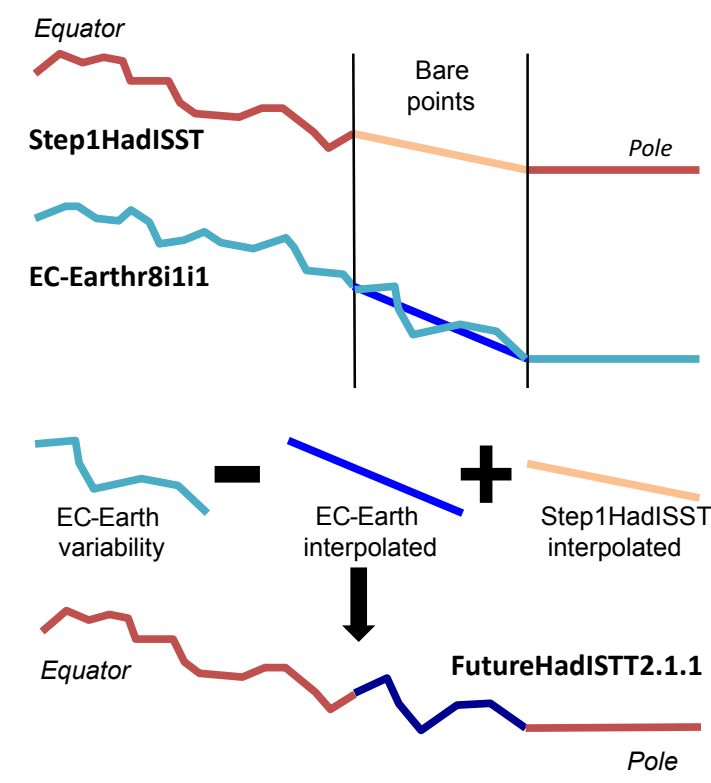

Figure 4. Scheme representing the methodology adopted to fill the "bare points", i.e. the points where sea ice has retreated in the CMIP5 EC-Earth RCP8.5 simulation. Each line represent a SST profile from the Equator to the pole.

tive variables (SMON). A few fields that are non-linear functions of the output (e.g. specific humidity) have been computed from the original $3 \mathrm{~h}$ output and then averaged at the required frequency in order to record them accurately. In addition to the atmospheric data, about $10 \mathrm{~TB}$ of oceanic output has been stored for PFC simulations. Data at daily and pentad frequency have been retained.

All the data, including raw output, post-processed data and restart files, have been archived on the tape archives of the Tivoli Storage Management Infrastructure of the LRZ.

\section{Results overview}

In this section we present a brief overview of the preliminary findings of the Climate SPHINX project. Considering that the number of diverse climate aspects that could be analysed in such a large dataset is large, we decided to present hereafter only a few selected features of the mean climate and its variability. For all the results presented - if not specified differently - the complete set of ensemble members available for the present-day climate (i.e. PDA experiments) has been used.

\subsection{Mean climate}

Although a detailed analysis of the mean climate in all the simulations performed would be excessively long to be included in the present work, we introduce a couple of figures showing the sensitivity to resolution and stochastic physics parameterisation of the climatology of precipitation (Fig. 5) and $200 \mathrm{hPa}$ zonal wind (Fig. 6). We compare the ensemble mean average fields of a low-resolution version (T255) with a high-resolution one (T799), in both its deterministic and stochastic configurations. Data have been interpolated on a common $2.5^{\circ} \times 2.5^{\circ}$ grid.

The precipitation model bias - shown in Fig. 5, with respect to Global Precipitation Climatology Project (GPCP) dataset (Huffman et al., 2001) - is especially strong in Indian Monsoon region, with an excess of precipitation from the Indian Ocean to the western Pacific. More generally, EC-Earth tends to underestimate the precipitation over the continents and overestimate it over the oceans. When the comparison is carried out between stochastic and deterministic configurations, it is possible to see that SPPT and SKEB neither improve nor deteriorate the climatology at both T255 and T799 resolutions. Conversely, a slightly more evident change is seen comparing the high and low resolutions; here T799 shows a widespread increase of the extratropical precipitation. But again, when it is evaluated against the model bias such changes are minor.

Impacts on the upper-tropospheric zonal wind field are clearer and they are shown in Fig. 6. The T255 version - compared against ECMWF ERA-Interim reanalysis (Dee et al., 2011) - shows too strong jets in both the hemispheres. The subtropical jet over Asia and the Pacific is also poleward displaced, while equatorial easterly jets are too weak. Again, stochastic physics bring minor changes, with a slightly stronger Atlantic jet, more penetrating over Europe. Conversely, the higher resolution leads to an overall weakening of the upper-tropospheric winds; this is especially true over North America and the Tibetan Plateau, suggesting that this change may be induced by the stronger surface drag caused by the more resolved (and thus higher) mean orography.

More generally, in these and other climatological fields (not shown) the impact of the two stochastic parameterisations and resolution appears to be small if compared to the model bias. Indeed, larger benefits from increasing resolution and stochastic physics are expected more in terms of variability rather than in terms of mean state (e.g. Dawson and Palmer, 2015; Wang et al., 2016; Christensen et al., 2017a).

Therefore, in the following sections we will focus on a few selected features of climate variability. We will investigate the improvements and/or deteriorations following resolution increase and including the SPPT and SKEB stochastic parameterisations of three different phenomena: the distribution of the intensity of tropical rainfall, the tropical variability related to the Madden-Julian Oscillation and the mid-latitude variability associated with atmospheric blocking.

\subsection{Tropical rainfall variability}

Climate models generally have too little tropical variability on timescales of several days (e.g. Hung et al., 2013). One aspect of the variability of particular interest is the oc- 

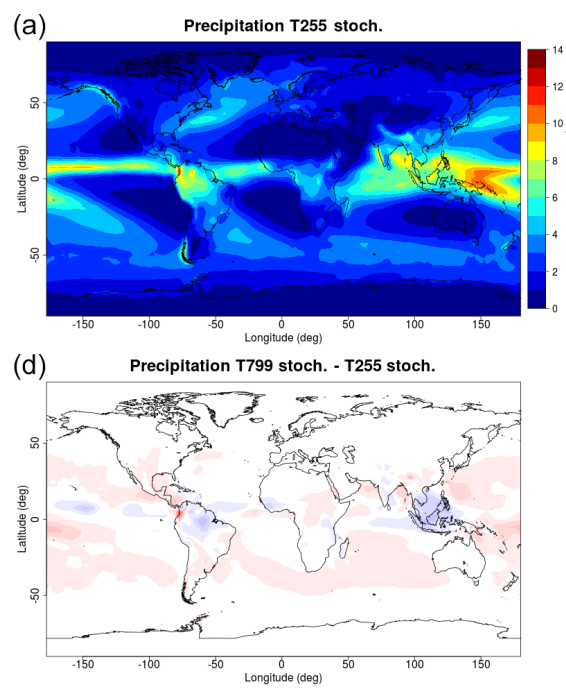

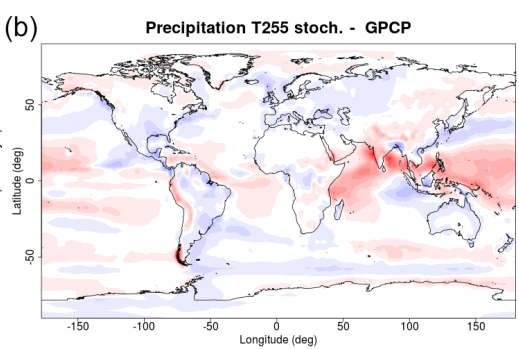

(e)

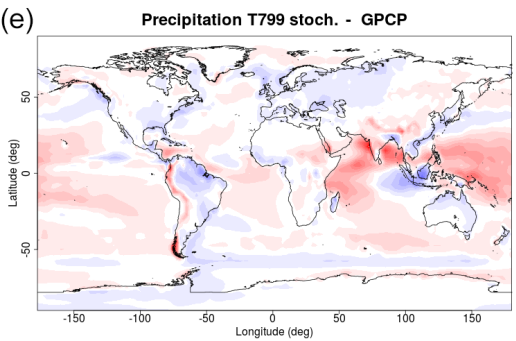

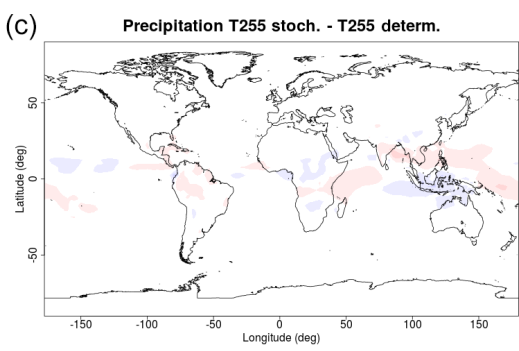

(f)

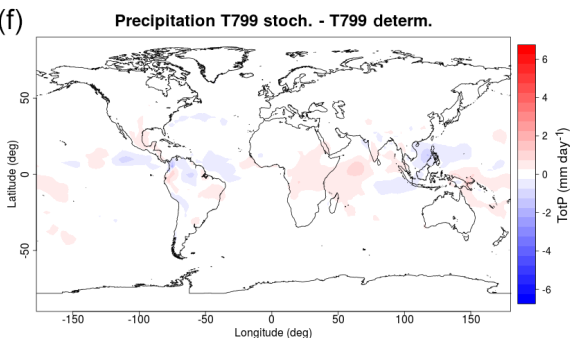

Figure 5. Left: (a) climatological ensemble mean precipitation for the PDA experiments (1979-2008) for T255 with stochastic physics. (d) T799 stochastic minus T255 stochastic precipitation. Centre: T255 (b) and T799 (e) precipitation bias with respect to GPCP. Right: stochastic minus deterministic climatological precipitation for T255 (c) and T799 (f).

(a)

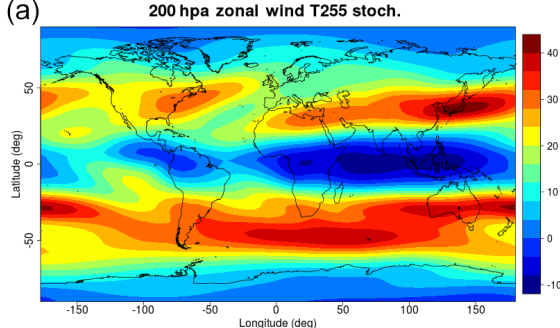

(d) $200 \mathrm{hpa}$ zonal wind T799 stoch. - T255 stoch.

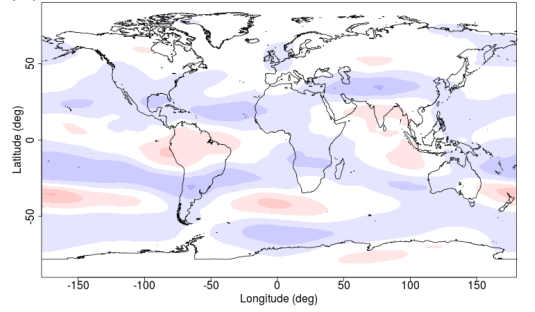

(b)

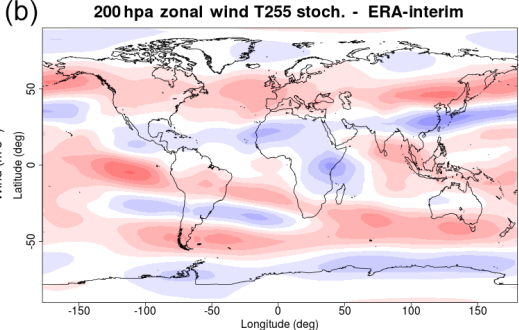

(e)

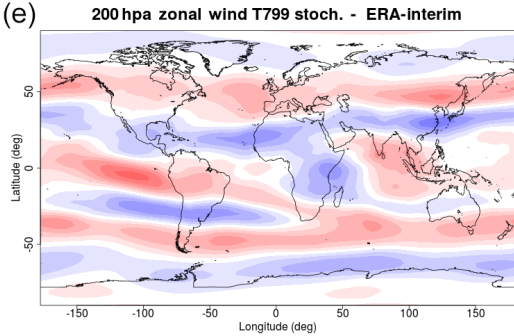

(c) $200 \mathrm{hpa}$ zonal wind T255 stoch. - T255 determ.

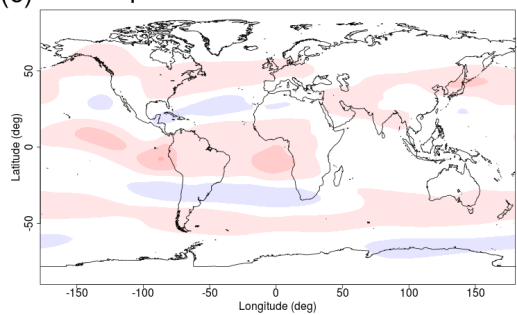

(f)

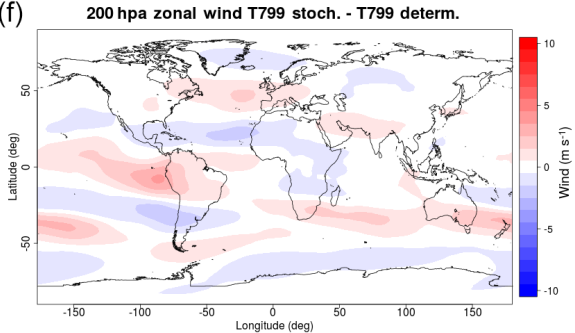

Figure 6. Same as Fig. 5 but for zonal wind at $200 \mathrm{hPa}$. Here bias is evaluated against ERA-Interim reanalysis.

currence of heavy-precipitation events, which can result in flooding, affect disease incidence and reduce crop yields (IPCC, 2015). Changes in the frequency of these events can also affect trends in total precipitation due to non-linearity in land-surface processes (Saeed et al., 2013).

Figure 7a shows the frequency distribution of daily-mean precipitation rates averaged over $2.5^{\circ} \times 2.5^{\circ}$ grid boxes between $10^{\circ} \mathrm{S}$ and $10^{\circ} \mathrm{N}$ over the period $1998-2008$ in data from GPCP, data from the Tropical Rainfall Measuring Mission (TRMM) 3B42 version 7 product (Huffman et al., 2007) and one ensemble member for each PDA run. Figure $7 \mathrm{~b}$ shows the ratio of the frequency in each rain rate interval as a fraction of that in GPCP for each resolution.
Vertical bars in Fig. 7 show the $95 \%$ confidence intervals of the frequencies associated with sampling uncertainty. These were calculated using a bootstrap method. For each dataset, a surrogate dataset was created by randomly sampling individual years of data with replacement. The frequency distribution of the surrogates and their frequency ratios with respect to the GPCP surrogate were calculated. This was repeated 1000 times to produce the distribution of the calculations associated with sampling uncertainty, from which the confidence intervals were derived.

At all resolutions, rain rates below $15 \mathrm{mmday}^{-1}$ occur too often in the model data, by about $50 \%$, and rain rates between 20 and $60 \mathrm{mmday}^{-1}$ occur too infrequently com- 
Frequency distribution of daily-mean total precip

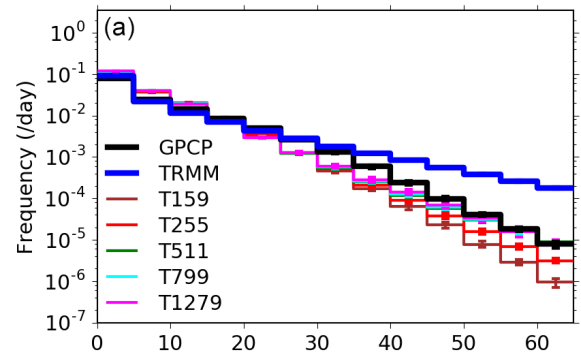

Frequency as fraction of that in GPCP
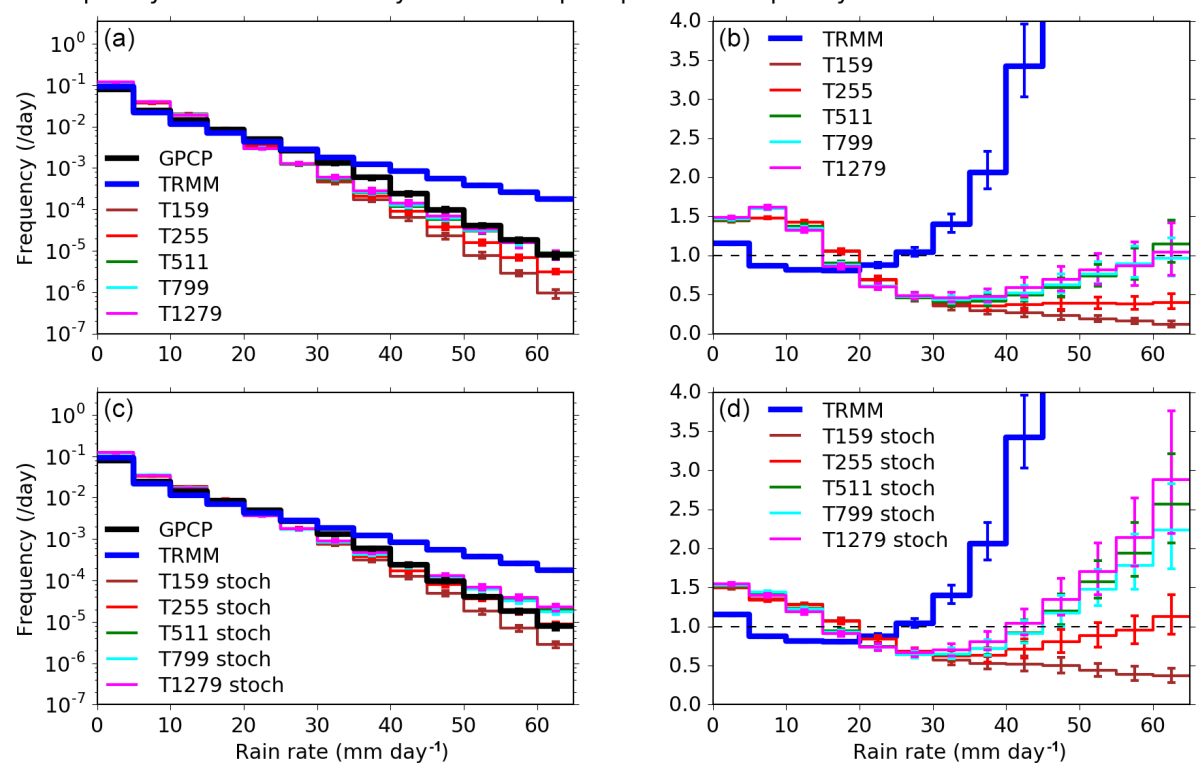

Figure 7. Panels (a) and (c) show the frequency of occurrence of daily-mean rain rates averaged over $2.5^{\circ} \times 2.5^{\circ}$ grid boxes between $10^{\circ} \mathrm{S}$ and $10^{\circ} \mathrm{N}$ in different datasets in $5 \mathrm{~mm} \mathrm{day}^{-1}$ intervals, with rates below $0.1 \mathrm{~mm}$ day ${ }^{-1}$ omitted. Panel (a) shows data for GPCP, TRMM and the deterministic Climate SPHINX PDA simulations and (c) shows the same for the PDA simulations with stochastic physics. Note that the vertical axis is logarithmic. Panels (b) and (d) show the rain rates in each simulation and TRMM as a fraction of that in GPCP for the deterministic and stochastic runs respectively. Horizontal dashed lines indicate a fraction of 1 , which would correspond to perfect agreement with GPCP. Vertical bars indicate the $95 \%$ confidence intervals. The frequency in (a) and (c) corresponds to that for an individual grid box, if all grid boxes were statistically equivalent. Data are shown for 1998-2008, the time period common to all datasets, for all ensemble members.

pared to both observational datasets. At rain rates near $30 \mathrm{~mm} \mathrm{day}^{-1}$, the simulated frequencies are between about 35 and $50 \%$ of the frequency in GPCP. At higher rain rates, the frequency differences between TRMM and GPCP become comparable in size to or larger than the differences between the modelled frequencies and the observational datasets. We do not know of a reason to strongly prefer one dataset over the other; therefore, we consider the model bias to be uncertain at these rain rates. The frequency of rain rates above $30 \mathrm{~mm} \mathrm{day}^{-1}$ in the T159 and T255 models are below about $40 \%$ of that in GPCP. At T511, T799 and T1279 the relative frequency difference compared to GPCP and TRMM decreases as the rain rate increases, and becomes comparable to that in GPCP in the $60-65 \mathrm{~mm} \mathrm{day}^{-1}$ interval, though still much smaller than that in TRMM. Therefore, increasing the model resolution from T159 to T511 improves the simulated frequency of heavy-rainfall events compared to observational datasets, with the further improvements caused by increasing the resolution to T799 or T1279 being considerably smaller.

Figure $7 \mathrm{c}, \mathrm{d}$ show the same data for the stochastic PDA runs. Stochastic physics has a similar effect at all resolutions. Frequencies of rain rates between 5 and $15 \mathrm{~mm} \mathrm{day}^{-1}$ are reduced by about $10 \%$ compared to those in the deterministic models, reducing the model bias. Frequencies above about $\sim 20 \mathrm{~mm} \mathrm{day}^{-1}$ are substantially increased, by a larger factor at larger rain rates, up to a factor of $\sim 2.5$ at rain rates around
$60 \mathrm{~mm} \mathrm{day}^{-1}$. This reduces the difference from GPCP and TRMM up to rain rates of $45 \mathrm{~mm} \mathrm{day}^{-1}$ at all resolutions.

The higher-resolution stochastic models have rain rate frequencies between those of GPCP and TRMM at rates above $45 \mathrm{~mm} \mathrm{day}^{-1}$, so they seem consistent with the observations given the observational uncertainty. The T255 stochastic model has rain rate frequencies closer to those in GPCP than any of the deterministic models in all but two of the $5 \mathrm{~mm} \mathrm{day}^{-1}$ rain rate intervals shown. One hypothesis to explain this effect is that the stochastic perturbations sometimes increase the moistening tendency of the air, so that it occurs more often that there is a high amount of water vapour in the air and heavier rain events can occur, and there is a compensating decrease in the frequency of moderate rain events.

Therefore, stochastic physics brings this aspect of the simulations into better agreement with observations, suggesting that including a representation of unresolved variability and model error is important for simulating the statistics of extreme tropical precipitation events.

\subsection{The Madden-Julian Oscillation variability}

The MJO is the dominant mode of variability in the tropical region on sub-seasonal timescales (Madden and Julian, 1994). It is characterised by a strong interaction between tropical convection and the large-scale environment, manifest as a coherent eastward propagating pattern of precipita- 

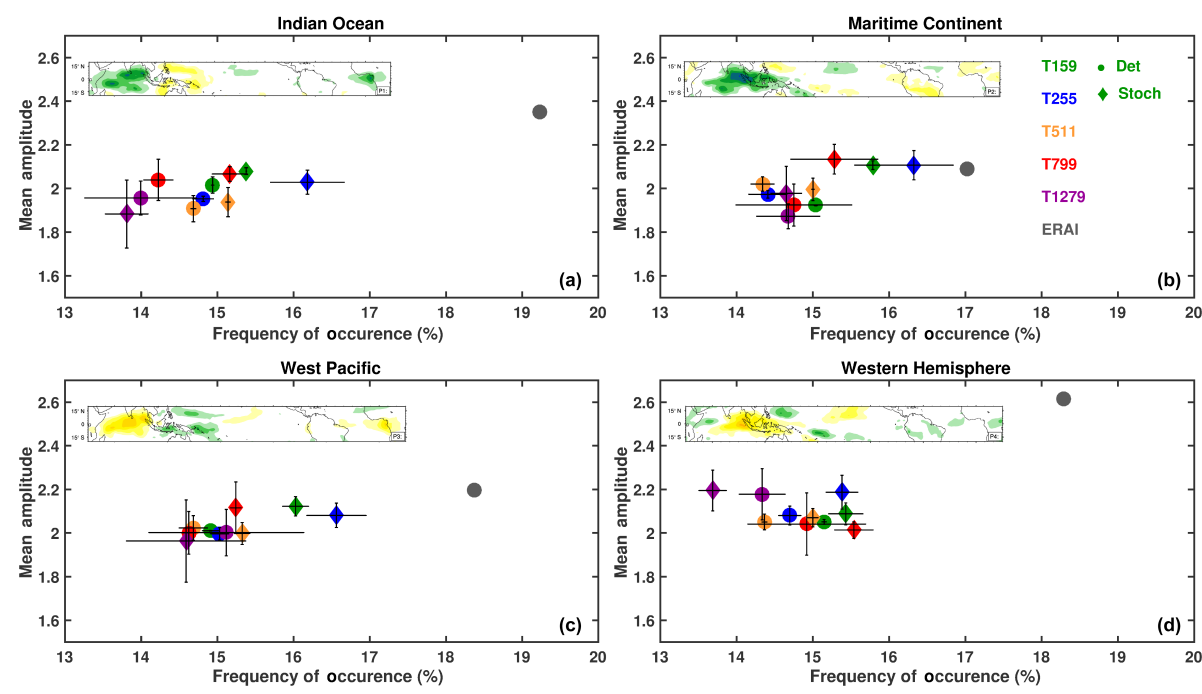

Figure 8. MJO frequency of occurrence vs. mean amplitude for the PDA experiments in the four different phases given the MJO amplitude to be $>1$. The four phases are classified as Indian Ocean (a), Maritime Continent (b), western Pacific (c) and western hemisphere (d), and their geographical location is shown by the boxes at the bottom of each panel with anomalous positive/negative precipitation patterns (green/yellow regions). Colours indicate the ensemble mean of the different resolutions as shown in the legend, where the circles are the deterministic runs and the diamonds the stochastic runs. ERA-Interim is reported in grey. Statistics are shown for the period 1980-2001. Error bars show the uncertainty range by providing the same statistic for periods half the length of the analysis.

tion followed by subsequent rainfall suppression (Khouider et al., 2011; Zhang et al., 2013; Kim et al., 2014; Raymond et al., 2015). It is a challenge for the current generation of global climate and weather models to represent the dynamics and thermodynamics of the MJO realistically (Slingo et al., 1996; Lin et al., 2006; Kim et al., 2009; Sperber et al., 2011; Klingaman et al., 2015).

Here we use the Wheeler and Hendon (2004) technique to identify the dominant modes of variability in zonal winds and outgoing longwave radiation (OLR) in these model runs. Combined Empirical Orthogonal Functions (CEOFs) of intraseasonal OLR, U850 (zonal winds at $850 \mathrm{hPa}$ ) and U200 (zonal winds at $200 \mathrm{hPa}$ ) are computed for each of the runs. The first two leading modes (Realtime Multivariate MJOs: RMM1 and RMM2) correspond to MJO signatures in the tropical wind field and OLR. The amplitude $A$ of the MJO is defined as

$A=\sqrt{(\mathrm{RMM} 1)^{2}+(\mathrm{RMM} 2)^{2}}$

and the phase $\Phi$ of the MJO is defined as

$\Phi=\tan ^{-1}\left(\frac{\mathrm{RMM} 2}{\mathrm{RMM} 1}\right)$.

MJO occurrence is defined when the MJO $A$ is $>1$. Conventionally, eight phases of the MJO are defined (Gottschalck et al., 2010). We reduce the eight phases to four phases respectively corresponding to the MJO being active in the Indian Ocean, Maritime Continent, Western Pacific and Western Hemisphere. We note that the Wheeler Hendon RMM index has been shown to be deficient in detecting
MJO events when large-scale circulation signals of the MJO are missing (Straub, 2013). Figure 8 shows the frequency of occurrence vs. the mean amplitude of the MJO in the four different regions around the tropics for all the different runs (colours) and for ECMWF ERA-Interim Reanalysis (grey; Dee et al., 2011) over the 1980-2001 period.

Overall the frequency of occurrence of the MJO in the different regions in the tropics for the different model resolutions is underestimated with respect to that of ERA-Interim. The MJO amplitude in the model simulations is lower than reanalysis over the Indian Ocean and the Western Hemisphere.

More importantly, increasing horizontal resolution does not seem to improve the representation of the phenomenon significantly. This may be explained considering that the simulation of the MJO in GCMs is influenced primarily by the representation of mesoscale dynamics and of convection. The simulation of mesoscale dynamics can be helped by increasing the resolution, while improvements in convection are driven by changes in physical parameterisations of the model. Yet, the coupling between the mesoscale dynamics and the convection is key for convectively coupled waves in the tropics (Raymond et al., 2015). Therefore, increasing resolution alone may not be sufficient to improve the simulation of the MJO.

Conversely, the stochastic physics parameterisation improves the MJO frequency in all regions at all resolutions but T1279. It must be noted that this latter run was done with only one ensemble member compared to the other runs with three or more ensemble members over the same period; 
therefore, a sampling error due to natural variability should be considered. Above all, the best results are obtained for the T255 with stochastic physics, suggesting that the tuning of the mean state of the model might play a relevant role for a better MJO simulation.

Additionally, the stochastic physics climate runs show an improvement in the representation of the MJO propagation over the Maritime Continent (not shown). The lack of propagation of the MJO over the Maritime Continent into the western Pacific region is a known problem in GCMs (Zhang et al., 2013). An improvement in the MJO propagation past the Maritime Continent due to SPPT has also been seen in the ECMWF seasonal forecasting system 4 (Weisheimer et al., 2014). Subramanian et al. (2017) also showed an improved MJO propagation and improved probabilistic prediction skill for the MJO in the ECMWF system 4 when SPPT is active as compared to runs without stochastic physics. Such improved propagation in stochastic runs indicates either that there is an impact of the stochastic physics on the mean state in the region or that the variability in the region helps maintain the intraseasonal signal. The reasons for the change in MJO representation due to stochastic physics will be explored further in a more detailed future study by the authors.

\subsection{Mid-latitude atmospheric blocking variability}

One of the most important challenges for the current generation of climate models is the simulation of atmospheric blocking (Anstey et al., 2013; Masato et al., 2013; DunnSigouin and Son, 2013; Davini and D'Andrea, 2016). Blocking is a recurrent weather pattern typically occurring in the Northern Hemisphere at the exit of the Atlantic and Pacific jet stream, more frequently during the winter season but observed throughout the year (Rex, 1950; Tibaldi and Molteni, 1990). It is characterised by a high-pressure, long-lasting low-vorticity anomaly that "blocks" the mid-latitude westerly flow, diverting synoptic disturbances poleward or equatorward (Tyrlis and Hoskins, 2008; Davini et al., 2012). A blocking event can last several days or even weeks, and it may be associated with cold spells in winter and heat waves in summer (Sillmann et al., 2011; Dole et al., 2011).

Blocking here is diagnosed using the simple index introduced by D'Andrea et al. (1998), an extension of the better known Tibaldi and Molteni (1990) index. This 1-D blocking index detects the reversal of the zonal flow measuring the geopotential height gradient at $500 \mathrm{hPa}$ at $60^{\circ} \mathrm{N}$, providing a binary blocking time series for each longitude. Although there is some evidence (e.g. Berner et al., 2012; Dawson and Palmer, 2015) that stochastic physics may improve the blocking simulation, with the current diagnostic no statistically significant difference emerges - even at low resolution - when comparing deterministic and stochastic simulations. Therefore, the two simulations are combined together to provide an unique ensemble.
The upper panel of Fig. 9 shows the blocking frequency for the ERA-Interim Reanalysis (black) and the ensemble mean of the different horizontal resolutions (colours) of PDA experiments over the December-January-February (DJF) period. The common negative bias over the Atlantic and Pacific basins is clearly evident. Increasing the horizontal resolution leads to benefits over both the basins, with marked improvements especially for the Atlantic; here, T799 and T1279 runs show values comparable to the reanalysis. The largest improvement is, however, seen upgrading from T255 to T511, where the bias - measured as the relative difference between the blocking frequency averaged between $10^{\circ} \mathrm{W}$ and $30^{\circ} \mathrm{E}-$ is reduced from the 18 to $3 \%$.

Those clear improvements in blocking frequency are interestingly reflected by a change in the mean state. A simple way to represent the flow variability is to highlight a few isopleths of geopotential height, as done in the lower panel of Fig. 9. Indeed, the higher-resolution models show a strengthened pattern of the dominant Northern Hemisphere planetary waves, with marked ridges over the Rockies and Europe. Especially, the former over the Rockies (Brayshaw et al., 2009) suggests an important role of orography resolution in the representation of the eddy-driven jet stream and, indirectly, of Euro-Atlantic blocking frequencies.

Indeed, the reduction of the bias following resolution increase for winter Atlantic blocking (and not for Pacific blocking) seems to be a common feature of several GCMs (Davini and D'Andrea, 2016; Schiemann et al., 2016). Such improvements have been associated with both better resolved transient eddy activity - which should sustain the blocking persistence (Shutts, 1983; Berckmans et al., 2013) - and with higher orography variance - which affects the mean state through planetary waves shaping (Jung et al., 2012; Berckmans et al., 2013). Conversely, Pacific blocking has been shown to be phenomenologically different (Pelly and Hoskins, 2003; Davini et al., 2012) and to be strongly affected by tropical dynamics (e.g. Renwick and Wallace, 1996); therefore, it is not surprising that the latter would be less affected by horizontal resolution changes.

A more detailed analysis of blocking and mid-latitude variability will be carried out by the authors in future studies.

\section{Conclusions}

In the present work we have described the scientific configuration and technical set-up/tuning of the EC-Earth Earth system model used for the Climate SPHINX project, which defines the SPHINX v1.0 protocol. More than 120 climate simulations have been produced making use of more than 20 million core hours and generating about $140 \mathrm{~TB}$ of postprocessed data. Climate SPHINX includes both present-day (PDA simulations, 1979-2008) and future scenario (FSA simulations, 2038-2068) atmosphere-only simulations according respectively to CMIP5 historical and RCP8.5 forc- 

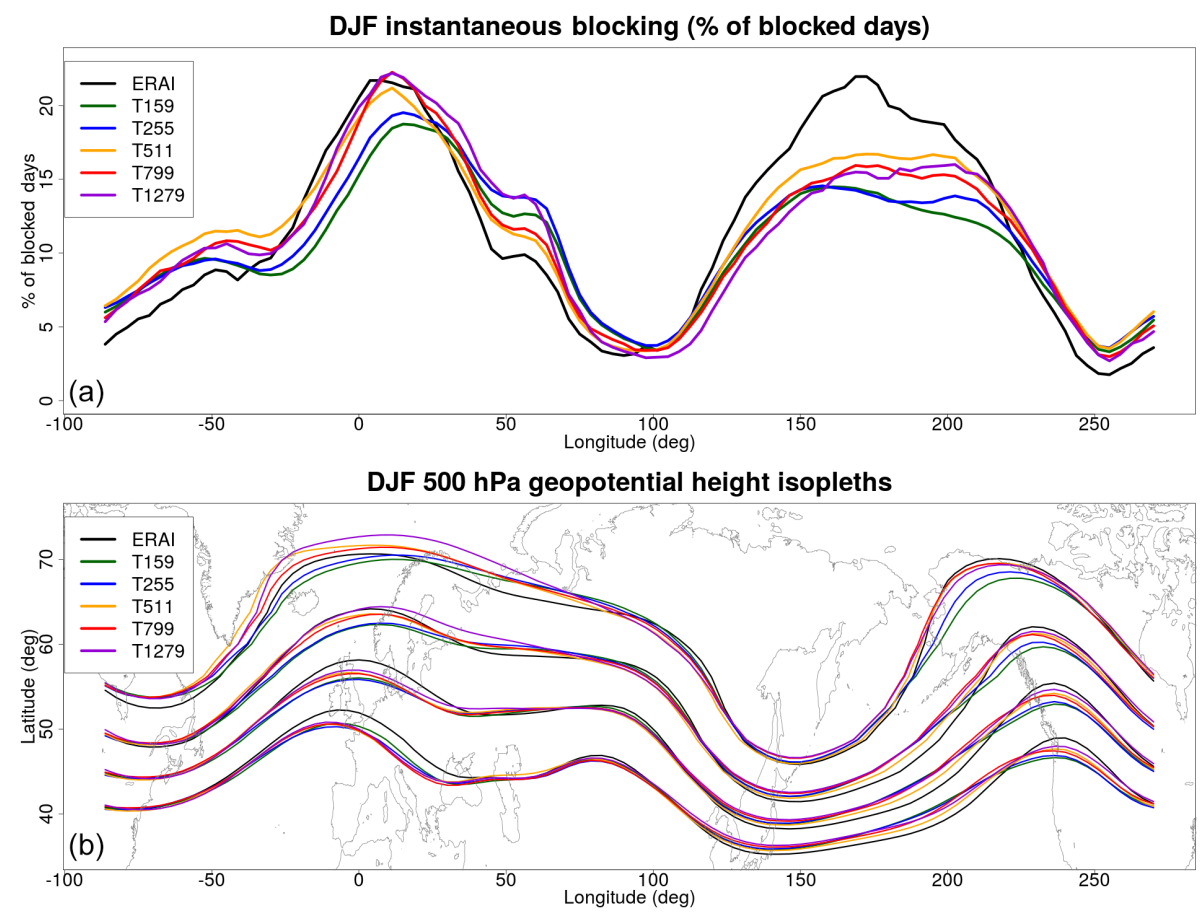

Figure 9. (a) Ensemble mean blocking frequencies following D'Andrea et al. (1998) for the different PDA experiments. Members of deterministic and stochastic experiments have been combined together for each resolution. ERA-Interim for the 1979-2008 period is shown as comparison in black. (b) December-January-February (DJF) climatological mean for geopotential height at $500 \mathrm{hPa}$ for the ensemble mean of PDA experiments. Only 5200, 5300, 5400 and $5500 \mathrm{~m}$ isopleths are reported.

ing. These have been run at five different horizontal resolutions - spanning from 125 to $16 \mathrm{~km}$ - with several ensemble members. Furthermore, a smaller set of transient coupled simulations (PFC simulations, 1850-2100) at T255 ORCA1 ( $\sim 80 \mathrm{~km}$ for the atmosphere and about $1^{\circ}$ for the ocean) has been run.

Each deterministic experiment included in Climate SPHINX has a counterpart where the sub-grid unresolved scales have been parameterised with two different stochastic physics schemes (namely the SPPT and SKEB schemes). This makes Climate SPHINX the first climate dataset that includes a large number of ensemble members with a stochastic parameterisation at different horizontal resolution; along with other high-resolution simulation campaigns such as UPSCALE (Mizielinski et al., 2014) or ATHENA (Kinter III et al., 2013), this demonstrates the ability of the climate community to exploit the more recent HPC machines.

Details on the tuning procedure (aimed at providing a correct radiation budget in the standard configuration T255) have been presented. Moreover, a comprehensive description of the methodology adopted for the creation of the presentday and future scenario SST and SIC (starting from the HadISST 2.1.1 dataset) has been described. A novel method aimed at estimating SST, where SICs have disappeared in future climate simulations, has been introduced.
More importantly, Climate SPHINX post-processed outputs are freely accessible to the climate community. This has been possible thanks to an EUDAT pilot project, which makes available a THREDDS server operational at CINECA from which data can be easily downloaded.

Preliminary results show the importance of both resolution and stochastic perturbations on the representation of the climate variability, although different phenomena show different sensitivities. Tropical rainfall variability seems to benefit from both increased horizontal resolution and stochastic parameterisation, whereas the Madden-Julian Oscillation shows improvements only when the stochastic perturbations are added. In general - in the tropics - applying stochastic schemes at low resolution leads to interesting improvements; on the other hand, increasing resolution beyond T511 does not seem to further improve the tropical variability.

Conversely, in the mid-latitudes, where atmospheric blocking frequencies were analysed, no statistical difference is found between stochastic and deterministic runs. Previous works (Berner et al., 2012; Dawson and Palmer, 2015) suggested that blocking regimes can benefit from stochastic schemes. We note that the simulations presented here are at a higher resolution than Berner et al. (2012) and have been analysed using a different metric to Dawson and Palmer (2015). Nevertheless, we must observe that our blocking diagnostic shows strong variability in the different ensemble 
members, suggesting that a single realisation may be not enough to capture the real sensitivity of this diagnostic to stochastic schemes. On the other hand, we found that increased horizontal resolution seems extremely important to decrease the blocking bias; in agreement with other recent works (Davini and D'Andrea, 2016; Schiemann et al., 2016) this is true especially over the Euro-Atlantic sector - where the T799 resolution $(\sim 25 \mathrm{~km})$ reduces it to negligible values - but not evident over the Pacific.

To summarise, the best improvements are observed on upgrading from T255 to T511, whereas minor improvements are observed using higher resolutions. However, while this resolution increase reduces the bias for the most of the phenomena here analysed, SPPT and SKEB schemes seem ineffective on some aspects (e.g. atmospheric blocking) but effective as much as resolution - and even more - on others (e.g. tropical precipitation variability); in the case of the MJO variability, stochastic schemes applied to the T255 model bring improvements larger than the ones associated with any resolution refinement.

However, we must remark that these results can be associated with the absence of specific tuning for both deterministic higher-resolution and stochastic configurations, which can affect the mean climate and consequently partially deteriorate the climate variability. Indeed, such tuning does not involve only the surface and TOA radiative fluxes but also some of the physical parameterisations of the climate model. Some schemes, e.g. deep and shallow convection parameterisations, may be satisfactory at coarse resolutions but may perform poorly at finer ones.

Given the similarities between the dynamical cores of climate models, since they are all based on a controlled discretisation of the same governing equations, we hope that the resolution sensitivity aspect of Climate SPHINX will be useful to the whole climate modelling community. On the other hand, several promising stochastic schemes exist, and the sensitivity of EC-Earth to SPPT and SKEB described here cannot be easily extrapolated to these alternative approaches. Nevertheless, considering that Climate SPHINX is the first large experiment where stochastic schemes are used massively on the climate time range, we hope that this work paves the way for other climate-oriented simulations aimed at investigating the impact of different stochastic schemes on climate variability.

Furthermore, Climate SPHINX focuses attention on the controversial choice between increasing resolution or increasing the size of ensembles - whilst keeping the same computing time available (e.g. Buizza et al., 1998). Indeed, running 30 years of one member at T1279 on the SuperMUC Petascale System costs about 1.4 million core hours; with the same amount of time it would be possible to run 9-10 simulations at T511. However, the benefits of the two pathways may be different, while a single member with $16 \mathrm{~km}$ resolution can provide local information at a topographic scale, which is useful, for instance, for hydrological models - par- ticularly in areas with complex topography; in contrast many ensemble members at $40 \mathrm{~km}$ resolution can provide a correct assessment of the natural variability, a key element for instance for mid-latitude climate (Deser et al., 2012; Kay et al., 2015). However, we must keep in mind that the computational constraints would become particularly relevant for coupled simulations, in which the computing time devoted to the oceanic model and - above all - to the spin-up of the coupled system will inflate considerably the number of core hours needed. Stochastic physics parameterisations, especially at lower resolution, seem able to provide an interesting alternative to tackle such controversy, improving model performance without increasing the nominal resolution and the overall computational cost.

Data availability. Post-processed data have been transferred from LRZ to CINECA via GridFTP, where they have been permanently stored. More importantly, free data accessibility to the climate user community is granted through a dedicated THREDDS Web Server hosted by CINECA (https://sphinx.hpc.cineca.it/thredds/ sphinx.html), where it is possible to browse and directly download Climate SPHINX data. Details on the data accessibility and on the Climate SPHINX project itself are available on the website of the project (http://www.to.isac.cnr.it/sphinx/). The set-up of this infrastructure for data sharing has been possible thanks to DATA SPHINX, an EUDAT data pilot project, which will allow long-term storage and sharing among a wide scientific user community of high-resolution climate model output data. DATA SPHINX aims to build a repository serving the climate change impact modelling community, providing selected variables at high temporal and spatial resolution, with a focus on climate extremes and the hydrological cycle in areas with complex orography.

Competing interests. The authors declare that they have no conflict of interest.

Acknowledgements. We acknowledge PRACE for awarding us access to resource SuperMUC based in Germany at the Leibniz Supercomputing Centre of the Bavarian Academy of Sciences and Humanities (LRZ) and to the Marconi HPC machine at CINECA, Italy. We thank the EUDAT (European Data Infrastructure) project for awarding us a data pilot project and CINECA for providing resources and technical assistance for the storage and distribution of the post-processed model output files. We thank Peter Bechtold and ECMWF for useful suggestions on the QBO tuning. Paolo Davini acknowledges the funding from the European Union's Horizon 2020 research and innovation programme COGNAC under the European Union Marie Skłodowska-Curie grant agreement no. 654942. Jost von Hardenberg and Paolo Davini acknowledge support by the Project of Interest NextDATA (MIUR PNR 2011-2013). Hannah M. Christensen, Stephan Juricke, Aneesh Subramanian, Peter A. G. Watson and Tim N. Palmer were supported under the European Research Council grant 291406 PESM. This study was supported by the project SPECS (grant agreement number 308378) funded by the European Commission 
Seventh Framework Research Programme. The authors also acknowledge support by the PRIMAVERA project, funded by the European Commission under grant agreement no. 641727 of the Horizon 2020 Research Programme. Jost von Hardenberg acknowledges support from the European Union's Horizon 2020 research and innovation programme under grant agreement no. 641816 (CRESCENDO) and thanks ECMWF for providing computing time in the framework of the special project SPITVONH.

Edited by: W. Hazeleger

Reviewed by: two anonymous referees

\section{References}

Anstey, J. A., Davini, P., Gray, L. J., Woollings, T. J., Butchart, N., Cagnazzo, C., Christiansen, B., Hardiman, S. C., Osprey, S. M., and Yang, S.: Multi-'model analysis of Northern Hemisphere winter blocking: Model biases and the role of resolution, J. Geophys. Res.-Atmos., 118, 3956-3971, 2013.

Arnold, H., Moroz, I., and Palmer, T.: Stochastic parametrizations and model uncertainty in the Lorenz'96 system, Philos. T. R. Soc. Lond, 371, 20110479, doi:10.1098/rsta.2011.0479, 2013.

Balsamo, G., Viterbo, P., Beljaars, A., van den Hurk, B., Hirschi, M., Betts, A. K., and Scipal, K.: A revised hydrology for the ECMWF model: Verification from field site to terrestrial water storage and impact in the Integrated Forecast System, J. Hydrometeorol., 10, 623-643, 2009.

Beljaars, A., Bechtold, P., Köhler, M., Morcrette, J.-J., Tompkins, A., Viterbo, P., and Wedi, N.: The numerics of physical parametrization, Proc. of ECMWF Seminar on Recent Developments in Numerical Methods for Atmosphere and Ocean Modelling, ECMWF, Reading, UK, 2004.

Bengtsson, L., Steinheimer, M., Bechtold, P., and Geleyn, J.-F.: A stochastic parametrization for deep convection using cellular automata, Q. J. Roy. Meteor. Soc., 139, 1533-1543, 2013.

Berckmans, J., Woollings, T., Demory, M.-E., Vidale, P.-L., and Roberts, M.: Atmospheric blocking in a high resolution climate model: influences of mean state, orography and eddy forcing, Atmos. Sci. Lett., 14, 34-40, 2013.

Berner, J., Shutts, G., Leutbecher, M., and Palmer, T.: A spectral stochastic kinetic energy backscatter scheme and its impact on flow-dependent predictability in the ECMWF ensemble prediction system, J. Atmos. Sci., 66, 603-626, 2009.

Berner, J., Jung, T., and Palmer, T.: Systematic model error: the impact of increased horizontal resolution versus improved stochastic and deterministic parameterizations, J. Climate, 25, 49464962, 2012.

Bouttier, F., Vié, B., Nuissier, O., and Raynaud, L.: Impact of stochastic physics in a convection-permitting ensemble, Mon. Weather Rev., 140, 3706-3721, 2012.

Brayshaw, D., Hoskins, B., and Blackburn, M.: The basic ingredients of the North Atlantic storm track, Part I: land-sea contrast and orography, J. Atmos. Sci., 66, 2539-2558, 2009.

Buizza, R., Petroliagis, T., Palmer, T., Barkmeijer, J., Hamrud, M., Hollingsworth, A., Simmons, A., and Wedi, N.: Impact of model resolution and ensemble size on the performance of an ensemble prediction system, Q. J. Roy. Meteor. Soc., 124, 1935-1960, 1998.
Christensen, H. M., Moroz, I. M., and Palmer, T. N.: Simulating Weather Regimes: impact of stochastic and perturbed parameter schemes in a simple atmospheric model, Clim. Dynam., 44, 2195-2214, 2015.

Christensen, H. M., Berner, J., Coleman, D., and Palmer, T. N.: Stochastic parametrisation and the El Niño-Southern Oscillation, J. Climate, 30, 17-38, 2017a.

Christensen, H. M., Dawson, A., and Palmer, T.: Constraining Stochastic Parametrisation Schemes using High-resolution Model Simulations, in preparation, 2017b.

D’Andrea, F., Tibaldi, S., Blackburn, M., Boer, G., Deque, M., Dix, M. R., Dugas, B., Ferranti, L., Iwasaki, T., Kitoh, A., Pope, V., Randall, D., Roeckner, E., Straus, D., Stern, W., den Dool, H. V., and Williamson, D.: Northern Hemisphere atmospheric blocking as simulated by 15 atmospheric general circulation models in the period 1979-1988, Clim. Dynam., 14, 383-407, 1998.

Davini, P. and D'Andrea, F.: Northern Hemisphere atmospheric blocking representation in Global Climate Models: twenty years of improvements?, J. Climate, 29, 8823-8840, doi:10.1175/JCLI-D-16-0242.1, 2016.

Davini, P., Cagnazzo, C., Gualdi, S., and Navarra, A.: Bidimensional diagnostics, variability and trends of Northern Hemisphere blocking, J. Climate, 25, 6996-6509, 2012.

Davini, P., Filippi, L., and von Hardenberg, J.: Tuning EC-Earth from v3.01 to v3.1, Tech. Rep. 01/14, CNR-ISAC, UOS Torino, 2014.

Dawson, A. and Palmer, T.: Simulating weather regimes: impact of model resolution and stochastic parameterization, Clim. Dynam., 44, 2177-2193, 2015.

Dawson, A., Palmer, T., and Corti, S.: Simulating regime structures in weather and climate prediction models, Geophys. Res. Lett., 39, L21805, doi:10.1029/2012GL053284, 2012.

Dee, D. P., Uppala, S. M., Simmons, A. J., Berrisford, P., Poli, P., Kobayashi, S., Andrae, U., Balmaseda, M. A., Balsamo, G., Bauer, P., Bechtold, P., Beljaars, A. C. M., van de Berg, I., Biblot, J., Bormann, N., Delsol, C., Dragani, R., Fuentes, M., Greer, A. J., Haimberger, L., Healy, S. B., Hersbach, H., Holm, E. V., Isaksen, L., Kallberg, P., Kohler, M., Matricardi, M., McNally, A. P., Mong-Sanz, B. M., Morcette, J.-J., Park, B.-K., Peubey, C., de Rosnay, P., Tavolato, C., Thepaut, J. N., and Vitart, F.: The ERAInterim reanalysis: Configuration and performance of the data assimilation system, Q. J. Roy. Meteorol. Soc., 137, 553-597, 2011.

Delworth, T., Rosati, A., Anderson, W., Adcroft, A., Balaji, V., Benson, R., Dixon, K., Griffies, S., Lee, H., Pacanowski, R., Vecchi, G., Wittenberg, A., Zeng, F., and Zhang, R.: Simulated climate and climate change in the GFDL CM2. 5 high-resolution coupled climate model, J. Climate, 25, 2755-2781, 2012.

Demory, M.-E., Vidale, P. L., Roberts, M. J., Berrisford, P., Strachan, J., Schiemann, R., and Mizielinski, M. S.: The role of horizontal resolution in simulating drivers of the global hydrological cycle, Clim. Dynam., 42, 2201-2225, 2014.

Deng, Q., Khouider, B., and Majda, A. J.: The MJO in a coarseresolution GCM with a stochastic multicloud parameterization, J. Atmos. Sci., 72, 55-74, 2015.

Deser, C., Phillips, A., Bourdette, V., and Teng, H.: Uncertainty in climate change projections: The role of internal variability, Clim. Dynam., 1-20, 2012. 
Dole, R., Hoerling, M., Perlwitz, J., Eischeid, J., Pegion, P., Zhang, T., Quan, X.-W., Xu, T., and Murray, D.: Was there a basis for anticipating the 2010 Russian heat wave?, Geophys. Res. Lett., 38, L06702, doi:10.1029/2010GL046582, 2011.

Dorrestijn, J., Crommelin, D. T., Siebesma, A. P., Jonker, H. J., and Selten, F.: Stochastic convection parameterization with Markov Chains in an intermediate-complexity GCM, J. Atmos. Sci., 73, 1367-1382, 2016.

Dunn-Sigouin, E. and Son, S.-W.: Northern Hemisphere blocking frequency and duration in the CMIP5 models, J. Geophys. Res.Atmos., 118, 1179-1188, 2013.

ECWMF: IFS cycle36r1, http://www.ecmwf.int/research/ifsdocs/ CY36r1/, European Center for Medium Range Forecast, 2009.

Gottschalck, J., Wheeler, M., Weickmann, K., Vitart, F., Savage, N., Lin, H., Hendon, H., Waliser, D., Sperber, K., Nakagawa, M., Prestrelo, C., Flatau, M., and Higgins, W.: A framework for assessing operational Madden-Julian oscillation forecasts: a CLIVAR MJO working group project, B. Am. Meteorol. Soc., 91, 1247-1258, doi:10.1175/2010BAMS2816.1, 2010.

Grell, G. A. and Freitas, S. R.: A scale and aerosol aware stochastic convective parameterization for weather and air quality modeling, Atmos. Chem. Phys., 14, 5233-5250, doi:10.5194/acp-145233-2014, 2014.

Haarsma, R. J., Roberts, M. J., Vidale, P. L., Senior, C. A., Bellucci, A., Bao, Q., Chang, P., Corti, S., Fuckar, N. S., Guemas, V., von Hardenberg, J., Hazeleger, W., Kodama, C., Koenigk, T., Leung, L. R., Lu, J., Luo, J.-J., Mao, J., Mizielinski, M. S., Mizuta, R., Nobre, P., Satoh, M., Scoccimarro, E., Semmler, T., Small, J., and von Storch, J.-S.: High Resolution Model Intercomparison Project (HighResMIP v1.0) for CMIP6, Geosci. Model Dev., 9, 4185-4208, doi:10.5194/gmd-9-4185-2016, 2016.

Hazeleger, W., Severijns, C., Semmler, T., Ştefăănescu, S., Yang, S., Wang, X., Wyser, K., Dutra, E., Baldasano, J. M., Bintanja, R., Bougeault, P., Caballero, R., Ekman, A., Christensen, J., van den Hurk, B., Jimenez, P., Jones, C., Kållberg, P., Koenigk, T., McGrath, R., Miranda, P., Van Noije, T., Palmer, T., Parodi, J., Schmith, T., Selten, F., Storelvmo, T., Sterl, A., Tapamo, H., Vancoppenolle, M., Viterbo, P., and Willén, U.: EC-Earth: A Seamless Earth-System Prediction Approach in Action, B. Am. Meteorol. Soc., 91, 1357-1363, doi:10.1175/2010BAMS2877.1, 2010.

Hazeleger, W., Wang, X., Severijns, C., Ştefănescu, S., Bintanja, R., Sterl, A., Wyser, K., Semmler, T., Yang, S., Van den Hurk, B., van Noije, T., van der Linden, E., and van der Wiel, K.: ECEarth V2.2: description and validation of a new seamless Earth system prediction model, Clim. Dynam., 39, 2611-2629, 2012.

Hersbach, H., Peubey, C., Simmons, A., Berrisford, P., Poli, P., and Dee, D.: ERA-20CM: a twentieth-century atmospheric model ensemble, Q. J. Roy. Meteor. Soc., 141, 2350-2375, 2015.

Huffman, G. J., Adler, R. F., Morrissey, M. M., Bolvin, D. T., Curtis, S., Joyce, R., McGavock, B., and Susskind, J.: Global precipitation at one-degree daily resolution from multisatellite observations, J. Hydrometeorol., 2, 36-50, 2001.

Huffman, G. J., Bolvin, D. T., Nelkin, E. J., Wolff, D. B., Adler, R. F., Gu, G., Hong, Y., Bowman, K. P., and Stocker, E. F.: The TRMM multisatellite precipitation analysis (TMPA): Quasiglobal, multiyear, combined-sensor precipitation estimates at fine scales, J. Hydrometeorol., 8, 38-55, 2007.
Hung, M.-P., Lin, J.-L., Wang, W., Kim, D., Shinoda, T., and Weaver, S. J.: MJO and convectively coupled equatorial waves simulated by CMIP5 climate models, J. Climate, 26, 6185-6214, 2013.

IPCC: Climate change 2014: impacts, adaptation, and vulnerability. Part B: regional aspects, Contribution of Working Group II to the Fifth Assessment Report of the Intergovernmental Panel on Climate Change, 2015.

Jung, T., Miller, M., Palmer, T., Towers, P., Wedi, N., Achuthavarier, D., Adams, J., Altshuler, E., Cash, B., Kinter, J., Marx, L., Stan, C., and Hodges, K.: High-resolution global climate simulations with the ECMWF model in Project Athena: Experimental design, model climate, and seasonal forecast skill, J. Climate, 25, 31553172, 2012.

Kay, J., Deser, C., Phillips, A., Mai, A., Hannay, C., Strand, G., Arblaster, J., Bates, S., Danabasoglu, G., Edwards, J., Holland, M., Kushner, P., Lamarque, J., Lawrence, D., Lindsay, K., Middleton, A., Munoz, E., Neale, R., Oleson, K., Polvani, L., and Vertenstein, M.: The Community Earth System Model (CESM) large ensemble project: A community resource for studying climate change in the presence of internal climate variability, B. Am. Meteorol. Soc., 96, 1333-1349, 2015.

Kennedy, J., Reyner, N., Millington, S. C., and Saunby, M.: The Met Office Hadley Centre sea ice and sea-surface temperature data set, version 2, part 2: Sea surface temperature analysis, in preparation, 2017.

Khouider, B., Biello, J., and Majda, A. J.: A stochastic multicloud model for tropical convection, Commun. Math. Sci., 8, 187-216, 2010.

Khouider, B., St-Cyr, A., Majda, A. J., and Tribbia, J.: The MJO and convectively coupled waves in a coarse-resolution GCM with a simple multicloud parameterization, J. Atmos. Sci., 68, 240-264, 2011.

Kim, D., Sperber, K., Stern, W., Waliser, D., Kang, I.-S., Maloney, E., Wang, W., Weickmann, K., Benedict, J., Khairoutdinov, M., Lee, M., Neale, R., Suarez, M., Thayer-Calder, K., and Zhang, G.: Application of MJO simulation diagnostics to climate models, J. Climate, 22, 6413-6436, 2009.

Kim, D., Xavier, P., Maloney, E., Wheeler, M., Waliser, D., Sperber, K., Hendon, H., Zhang, C., Neale, R., Hwang, Y.-T., and Liu, H.: Process-oriented MJO simulation diagnostic: Moisture sensitivity of simulated convection, J. Climate, 27, 5379-5395, 2014.

Kinter III, J., Cash, B., Achuthavarier, D., Adams, J., Altshuler, E., Dirmeyer, P., Doty, B., Huang, B., Jin, E., Marx, L., and Manganello, J., Stan, C., Wakefield, T., Palmer, T., Hamrud, M., Jung, T., Miller, M., Towers, P., Wedi, N., Satoh, M., Tomita, H., Kodama, C., Nasuno, T., Oouchi, K., Yamada, Y., Taniguchi, H., Andrews, P., Baer, T., Ezell, M., Halloy, C., John, D., Loftis, B., Mohr, R., and Wong, K.: Revolutionizing climate modeling with Project Athena: A multi-institutional, international collaboration, B. Am. Meteorol. Soc., 94, 231-245, 2013.

Klingaman, N. P., Woolnough, S. J., Jiang, X., Waliser, D., Xavier, P. K., Petch, J., Caian, M., Hannay, C., Kim, D., Ma, H.-Y., Merryfield, W. J., Miyakawa, T., Pritchard, M., Ridout, J. A., Roehrig, R., Shindo, E., Vitart, F., Wang, H., Cavanaugh, N. R., Mapes, B. E., Shelly, A., and Zhang, G. J.: Vertical structure and physical processes of the Madden-Julian oscillation: Linking hindcast fidelity to simulated diabatic heating and moistening, J. Geophys. Res.-Atmos., 120, 4690-4717, 2015. 
Leutbecher, M., Lock, S.-J., Ollinaho, P., Lang, S. T. K., Balsamo, G., Bechtold, P., Bonavita, M., Christensen, H. M., Diamantakis, M., Dutra, E., English, S., Fisher, M., Forbes, R. M., Goddard, J., Haiden, T., Hogan, R. J., Juricke, S., Lawrence, H., MacLeod, D., Magnusson, L., Malardel, S., Massart, S., Sandu, I., Smolarkiewicz, P. K., Subramanian, A., Vitart, F., Wedi, N., and Weisheimer, A.: Stochastic representations of model uncertainties at ECMWF: State of the art and future vision, Q. J. Roy. Meteor. Soc., in preparation, 2017.

Lin, J.-L., Kiladis, G. N., Mapes, B. E., Weickmann, K. M., Sperber, K. R., Lin, W., Wheeler, M. C., Schubert, S. D., Del Genio, A., Donner, L. J., Emori, S., Gueremy, J., Hourdin, F., Rasch, P., Roeckner, E., and Scinocca, J.: Tropical intraseasonal variability in 14 IPCC AR4 climate models, Part I: Convective signals, J. Climate, 19, 2665-2690, 2006.

Lin, J. W.-B. and Neelin, J. D.: Influence of a stochastic moist convective parameterization on tropical climate variability, Geophy. Res. Lett., 27, 3691-3694, 2000.

Lin, J. W.-B. and Neelin, J. D.: Toward stochastic deep convective parameterization in general circulation models, Geophy. Res. Lett., 30, 1162, doi:10.1029/2002GL016203, 2003.

Lu, J., Chen, G., Leung, L. R., Burrows, D. A., Yang, Q., Sakaguchi, K., and Hagos, S.: Toward the dynamical convergence on the jet stream in aquaplanet AGCMs, J. Climate, 28, 6763-6782, 2015.

Madden, R. A. and Julian, P. R.: Observations of the 40-50-day tropical oscillation-A review, Mon. Weather Rev., 122, 814-837, 1994.

Madec, G.: NEMO ocean engine, Tech. rep., Institut Pierre-Simon Laplace (IPSL), 2008.

Masato, G., Hoskins, B. J., and Woollings, T.: Winter and Summer Northern Hemisphere blocking in CMIP5 models, J. Climate, 26, 7044-7059, 2013.

Mauritsen, T., Stevens, B., Roeckner, E., Crueger, T., Esch, M., Giorgetta, M., Haak, H., Jungclaus, J., Klocke, D., Matei, D., Mikolajewicz, U., Notz, D., Pincus, R., Schmidt, H., and Tomassini, L.,: Tuning the climate of a global model, J. Adv. Model. Earth Sys., 4, M00A01, doi:10.1029/2012MS000154, 2012.

Mizielinski, M. S., Roberts, M. J., Vidale, P. L., Schiemann, R., Demory, M.-E., Strachan, J., Edwards, T., Stephens, A., Lawrence, B. N., Pritchard, M., Chiu, P., Iwi, A., Churchill, J., del Cano Novales, C., Kettleborough, J., Roseblade, W., Selwood, P., Foster, M., Glover, M., and Malcolm, A.: High-resolution global climate modelling: the UPSCALE project, a large-simulation campaign, Geosci. Model Dev., 7, 1629-1640, doi:10.5194/gmd-71629-2014, 2014.

Mizuta, R., Adachi, Y., Yukimoto, S., and Kusunoki, S.: Estimation of future distribution of sea surface temperature and sea ice using CMIP3 multi-model ensemble mean, Technical Report of the Meteorological Research Institute, 28 pp., 2008.

Moss, R., Edmonds, J., Hibbard, K., Manning, M., Rose, S., van Vuuren, D., Carter, T., Emori, S., Kainuma, M., Kram, T., Meehl, G. A., Mitchell, J. F. B., Nakicenovic, N., Riahi, K., Smith, S. J., Stouffer, R. J., Thomson, A. M., Weyant, J. P., and Wilbanks, T. J.: The next generation of scenarios for climate change research and assessment, Nature, 463, 747-756, 2010.

Ollinaho, P., Lock, S.-J., Leutbecher, M., Bechtold, P., Beljaars, A., Bozzo, A., Forbes, R. M., Haiden, T., Hogan, R. J., and Sandu, I.: Towards process-level representation of model uncertainties:
Stochastically perturbed parametrisations in the ECMWF ensemble, Q. J. Roy. Meteor. Soc., 143, 408-422, doi:10.1002/qj.2931, 2016.

Palmer, T.: Towards the probabilistic Earth-system simulator: a vision for the future of climate and weather prediction, Q. J. Roy. Meteor. Soc., 138, 841-861, 2012.

Palmer, T., Buizza, R., Doblas-Reyes, F., Jung, T., Leutbecher, M., Shutts, G., Steinheimer, M., and Weisheimer, A.: Stochastic parametrization and model uncertainty, European Centre for Medium-Range Weather Forecasts, 2009.

Peatman, S. C., Matthews, A. J., and Stevens, D. P.: Propagation of the Madden-Julian Oscillation and scale interaction with the diurnal cycle in a high-resolution GCM, Clim. Dynam., 45, 29012918, 2015.

Pelly, J. and Hoskins, B.: A New Perspective on Blocking, J. Atmos. Sci., 60, 743-755, 2003.

Plant, R. and Craig, G. C.: A stochastic parameterization for deep convection based on equilibrium statistics, J. Atmos. Sci., 65, 87-105, 2008.

Raymond, D., Fuchs, Ž., Gjorgjievska, S., and Sessions, S.: Balanced dynamics and convection in the tropical troposphere, J. Adv. Model. Earth Sys., 7, 1093-1116, 2015.

Renwick, J. and Wallace, J.: Relationships between North Pacific wintertime blocking, El Niño, and the PNA pattern, Mon. Weather Rev., 124, 2071-2076, 1996.

Rex, D.: Blocking action in the middle troposphere and its effect upon regional climate: I. An aerological study of blocking action, Tellus, 2, 196-211, 1950.

Saeed, F., Haensler, A., Weber, T., Hagemann, S., and Jacob, D.: Representation of extreme precipitation events leading to opposite climate change signals over the Congo basin, Atmosphere, 4, 254-271, 2013.

Sakradzija, M., Seifert, A., and Dipankar, A.: A stochastic scaleaware parameterization of shallow cumulus convection across the convective gray zone, J. Adv. Model. Earth Sys., 8, 786-812, doi:10.1002/2016MS000634, 2016.

Sanchez, C., Williams, K. D., and Collins, M.: Improved stochastic physics schemes for global weather and climate models, Q. J. Roy. Meteor. Soc., 142, 147-159, 2016.

Schiemann, R., Demory, M.-E., Shaffrey, L. C., Strachan, J., Vidale, P. L., Mizielinski, M. S., Roberts, M. J., Matsueda, M., Wehner, M. F., and Jung, T.: The resolution sensitivity of Northern Hemisphere blocking in four 25-km atmospheric global circulation models, J. Climate, 30, 337-358, doi:10.1175/JCLI-D16-0100.1, 2016.

Shutts, G.: The propagation of eddies in diffluent jetstreams: eddy vorticity forcing of blocking flow fields, Q. J. Roy. Meteor. Soc., 109, 737-761, 1983.

Shutts, G.: A kinetic energy backscatter algorithm for use in ensemble prediction systems, Q. J. Roy. Meteor. Soc., 131, 3079-3102, 2005.

Shutts, G. and Pallarès, A. C.: Assessing parametrization uncertainty associated with horizontal resolution in numerical weather prediction models, Philos. T. R. Soc. A, 372, 20130284, doi:10.1098/rsta.2013.0284, 2014.

Shutts, G. and Palmer, T.: Convective forcing fluctuations in a cloud-resolving model: Relevance to the stochastic parameterization problem, J. Climate, 20, 187-202, 2007. 
Sillmann, J., Croci-Maspoli, M., Kallache, M., and Katz, R. W.: Extreme Cold Winter Temperatures in Europe under the Influence of North Atlantic Atmospheric Blocking, J. Climate, 24, 58995913, 2011.

Slingo, J., Sperber, K., Boyle, J., Ceron, J.-P., Dix, M., Dugas, B., Ebisuzaki, W., Fyfe, J., Gregory, D., Gueremy, J.-F., Hack, J., Harzallah, A., Inness, P., Kitoh, A., Lau, W. K.-M., McAvaney, B., Madden, R., Matthews, A., Palmer, T. N., Parkas, C.-K., Randall, D., and Renno, N.: Intraseasonal oscillations in 15 atmospheric general circulation models: results from an AMIP diagnostic subproject, Clim. Dynam., 12, 325-357, 1996.

Sperber, K., Slingo, J., and Inness, P.: Modeling intraseasonal variability, in: Intraseasonal Variability of the Atmosphere-Ocean Climate System, p. 613, 2011.

Straub, K. H.: MJO initiation in the real-time multivariate MJO index, J. Climate, 26, 1130-1151, 2013.

Subramanian, A., Weisheimer, A., Palmer, T., Vitart, F., and Bechtold, P.: Impact of stochastic physics on tropical precipitation in the coupled ECMWF model, Q. J. Roy. Meteor. Soc., 143, 852865, 2017.

Taylor, K., Stouffer, R., and Meehl, G.: An overview of CMIP5 and the experiment design, B. Am. Meteorol. Soc., 93, 485-498, doi:10.1175/BAMS-D-11-00094.1, 2012.

Tibaldi, S. and Molteni, F.: On the operational predictability of blocking, Tellus A, 42, 343-365, 1990.

Titchner, H. A. and Rayner, N. A.: The Met Office Hadley Centre sea ice and sea surface temperature data set, version 2: 1 . Sea ice concentrations, J. Geophys. Res.-Atmos., 119, 2864-2889, 2014.

Tyrlis, E. and Hoskins, B.: The Morphology of Northern Hemisphere Blocking, J. Atmos. Sci., 65, 1653-1665, 2008.

Valcke, S.: The OASIS3 coupler: a European climate modelling community software, Geosci. Model Dev., 6, 373-388, doi:10.5194/gmd-6-373-2013, 2013.
Vancoppenolle, M., Bouillon, S., Fichefet, T., Goosse, H., Lecomte, O., Morales Maqueda, M., and Madec, G.: LIM, The Louvain-laNeuve sea Ice Model, Notes du Pôle de modélisation, 2012.

van Oldenborgh, G. J., Doblas-Reyes, F. J., Wouters, B., and Hazeleger, W.: Decadal prediction skill in a multi-model ensemble, Clim. Dynam., 38, 1263-1280, 2012.

Wang, Y., Zhang, G. J., and Craig, G. C.: Stochastic convective parameterization improves the simulation of tropical precipitation variability in the NCAR CAM5, Geophys. Res. Lett., 43, 6612 6619, doi:10.1002/2016GL069818, 2016.

Weisheimer, A., Corti, S., Palmer, T., and Vitart, F.: Addressing model error through atmospheric stochastic physical parametrizations: impact on the coupled ECMWF seasonal forecasting system, Philos. T. R. Soc. A, 372, 20130290, doi:10.1098/rsta.2013.0290, 2014.

Wheeler, M. C. and Hendon, H. H.: An all-season real-time multivariate MJO index: Development of an index for monitoring and prediction, Mon. Weather Rev., 132, 1917-1932, 2004.

Wild, M., Folini, D., Schär, C., Loeb, N., Dutton, E. G., and KönigLanglo, G.: The global energy balance from a surface perspective, Clim. Dynam., 40, 3107-3134, 2013.

Yonehara, H. and Ujiie, M.: Stochastic physics scheme for model uncertainties in the JMA one-week ensemble prediction system, WMO CAS/JSC WGNE, 2011.

Zappa, G., Shaffrey, L. C., and Hodges, K. I.: The ability of CMIP5 models to simulate north atlantic extratropical cyclones, J. Climate, 26, 5379-5396, 2013.

Zhang, C., Gottschalck, J., Maloney, E. D., Moncrieff, M. W., Vitart, F., Waliser, D. E., Wang, B., and Wheeler, M. C.: Cracking the MJO nut, Geophys. Res. Lett., 40, 1223-1230, 2013. 